\title{
Modeling of ATP-Mediated Signal Transduction and Wave Propagation in Astrocytic Cellular Networks
}

\author{
Michail Stamatakis, Nikos V. Mantzaris \\ Department of Chemical and Biomolecular Engineering, Rice University, Houston, \\ TX 77005, USA \\ * Corresponding author. \\ Department of Chemical and Biomolecular Engineering, Rice University, Houston, \\ TX 77005, USA. \\ Phone: $+1-713-348-2955$ \\ Fax: $+1-713-348-5478$ \\ e-mail:nman@rice.edu
}

\begin{abstract}
Astrocytes, a special type of glial cells, were considered to have supporting role in information processing in the brain. However, several recent studies have shown that they can be chemically stimulated by neurotransmitters, and use a form of signaling, in which ATP acts as an extracellular messenger. Pathological conditions, such as spreading depression, have been linked to abnormal range of wave propagation in astrocytic cellular networks. Nevertheless, the underlying intra- and inter-cellular signaling mechanisms remain unclear. Motivated by the above, we constructed a model to understand the relationship between single-cell signal transduction mechanisms and wave propagation and blocking in astrocytic networks. The model incorporates ATP-mediated $\mathrm{IP}_{3}$ production, the subsequent $\mathrm{Ca}^{2+}$ release from the ER through $\mathrm{IP}_{3} \mathrm{R}$ channels and ATP release into the extracellular space. For the latter, two hypotheses were tested: $\mathrm{Ca}^{2+}$ - or $\mathrm{IP}_{3}$-dependent ATP release. In the first case, single astrocytes can exhibit excitable behavior and frequency-encoded oscillations. Homogeneous, one-dimensional astrocytic networks can propagate waves with infinite range, while in two dimensions, spiral waves can be generated. However, in the $\mathrm{IP}_{3}$-dependent ATP release case, the specific coupling of the driver ATP-IP 3
\end{abstract}


system with the driven $\mathrm{Ca}^{2+}$ subsystem leads to one- and two-dimensional wave patterns with finite range of propagation.

KEYWORDS astrocytes, ATP wave, $\mathrm{Ca}^{2+}$ wave, spiral wave, wave blocking, ATP release 


\section{INTRODUCTION}

The nervous system consists of two types of cells: the neurons, which are responsible for signal propagation and information processing and the glial cells which exist in between the neurons (Brodal, 1998). A special type of glial cells, which will be the focus of our modeling studies, are the so-called astrocytes. Before 1990, astrocytes were considered to have just supporting role for the neurons, by holding them together and supplying nutrients to them (Haydon, 2001; Nedergaard et al., 2003). This viewpoint was mainly due to the stimulation techniques that were employed: astrocytes are not electrically excitable like the neurons, and studies in which electrical stimuli were applied to them could not reveal their role (Haydon, 2001). One early exception came from the studies of Orkand et al. who showed that neuronal activity results in membrane depolarization of astrocytes (Orkand et al., 1966).

Major insights into the possible role of astrocytes in the mammalian brain have started to appear in the literature in the early 90 's, when new experimental techniques became available (ion-sensitive fluorescent indicators, quantitative imaging techniques, patch clamp recordings from brain slices, confocal microscopy) (Haydon, 2001). The milestone paper by Cornel-Bell showed that glutamate can induce $\mathrm{Ca}^{2+}$ waves in astrocytic cultures (Cornell-Bell et al., 1990). Many subsequent studies showed that astrocytes use a form of signaling to communicate with each other and with neighboring neurons. Thus, a focal stimulus applied to a specific cell, not only initiates a response from that cell, but it also results in a $\mathrm{Ca}^{2+}$ wave which is then propagated to the culture (Cornell-Bell et al., 1990). Wave propagation also occurs in situ in slices of the hippocampus (Nett et al., 2002) and the thalamus (Parri et al., 2001). The wave can subsequently reach and stimulate neurons (Nedergaard, 1994; 
Parpura et al., 1994). Finally, it has been suggested that abnormal wave propagation in astrocytic tissues is linked to pathological conditions such as spreading depression, migraine (Leibowitz, 1992; Nedergaard et al., 2003) and epilepsy (D'Ambrosio, 2004). These findings led to a reconsideration of the role of astrocytes in the brain. Astrocytes were no longer viewed as passive bystanders in the synapse, but as active components playing their role in information processing and signal propagation (Nedergaard et al., 2003).

At the single-cell level, it was discovered that astrocytes can modulate their intracellular calcium concentration when stimulated with a neurotransmitter, such as glutamate (Cornell-Bell et al., 1990) or ATP (Cotrina et al., 1998; Guthrie et al., 1999; Wang et al., 2000). The types of behavior that can be observed after stimulation involve a rapid increase in the cytosolic $\mathrm{Ca}^{2+}$ concentration followed by a sustained plateau phase, rapid increase in the cytosolic $\mathrm{Ca}^{2+}$ concentration which finally returns to the basal levels, and $\mathrm{Ca}^{2+}$ oscillations that persist for several minutes (Salm and McCarthy, 1990). There are several mechanisms by which the cell can increase its cytosolic $\mathrm{Ca}^{2+}$. They include $\mathrm{Ca}^{2+}$ influx from the extracellular space or efflux from intracellular $\mathrm{Ca}^{2+}$ stores, such as the endoplasmic reticulum (ER) and the mitochondria. However, it is considered that the main mechanism by which the $\mathrm{Ca}^{2+}$ concentration is modulated in astrocytes, involves an $\mathrm{IP}_{3}$-dependent pathway, which results in the release of $\mathrm{Ca}^{2+}$ through channels on the surface of the ER.

This pathway is schematically shown in Fig. 1. Astrocytes are known to express a variety of purine receptors (P2Y) (Nedergaard et al., 2003). When a neurotransmitter, such as ATP, binds to these receptors in the membrane of the cell, phospholipase C (PLC) is activated through a G-protein coupled pathway. The activated PLC catalyzes the conversion of phosphatidylinositol 4,5-bisphosphate $\left(\operatorname{PtdIns}(4,5) \mathrm{P}_{2}, \mathrm{PIP}_{2}\right)$ into 
inositol 1,4,5-trisphosphate $\left(\operatorname{Ins}(1,4,5) \mathrm{P}_{3}, \mathrm{IP}_{3}\right)$ and diacylglycerol (DAG) (Berridge et al., 1998). $\mathrm{IP}_{3}$ binds synergistically with $\mathrm{Ca}^{2+}$ ions to the $\mathrm{IP}_{3}$ receptor $\left(\mathrm{IP}_{3} \mathrm{R}\right)$ on the membrane of the ER and opens channels, through which $\mathrm{Ca}^{2+}$ is released into the cytosol. There exist also ATPase pumps on the surface of the ER which return $\mathrm{Ca}^{2+}$ back into the ER (Carafoli, 2002). There are two reasons for this: first the $\mathrm{Ca}^{2+}$ concentration in the cytosol must be kept low for it to function as an effective messenger and second, prolonged high cytosolic concentration of $\mathrm{Ca}^{2+}$ will signal cell death. Transient elevations of intracellular $\mathrm{Ca}^{2+}$ concentration are only one consequence of astrocytic stimulation. Stimulated astrocytes are also capable of glutamate uptake (Anderson and Swanson, 2000) as well as release of glutamate (Innocenti et al., 2000), ATP (Newman, 2001) and $\mathrm{Ca}^{2+}$ to the extracellular space (Goldman et al., 1994; Holgado and Beauge, 1995).

Moreover, the neurotransmitter- or mechanically- or electrically-induced $\mathrm{Ca}^{2+}$ responses do not remain local; they spread over a coupled network of astrocytes in the form of a calcium wave, which is characterized by a finite range of propagation in both space and time. Intercellular $\mathrm{Ca}^{2+}$ waves were first reported by Cornell-Bell et al. (1990), who described propagated increases in intracellular $\mathrm{Ca}^{2+}$ in astrocytes from rat hippocampus in response to bath application of glutamate. Glutamate-induced $\mathrm{Ca}^{2+}$ waves were also studied in rat and human hippocampal astrocytes in many other experiments (Cornell-Bell and Finkbeiner, 1991; Finkbeiner, 1992; Kim et al., 1994; Lee et al., 1995). Mechanically-induced intercellular $\mathrm{Ca}^{2+}$ waves were also observed in cortical mixed glia (Charles, 1994; Charles et al., 1991; Charles et al., 1993), C6 glioma cells (Charles et al., 1992), cortical astrocytes (Enkvist and McCarthy, 1992), oligodendrocytes (Takeda et al., 1995) and striatal astrocytes (Venance et al., 1997; 1995). In addition, electrical stimuli evoked intercellular $\mathrm{Ca}^{2+}$ waves in hippocampal 
slice cultures (Dani et al., 1992), in cortical astrocyte-neuron cultures (Parpura et al., 1994), in hippocampal astrocyte-neuron cultures (Hassinger et al., 1995) and in rat cortical astrocytes (Guthrie et al., 1999). Finally, local astrocytic stimulation with ATP was also found to result in $\mathrm{Ca}^{2+}$ wave propagation in the acutely isolated retina (Newman, 2001; Newman and Zahs, 1997). Radial, as well as spiral $\mathrm{Ca}^{2+}$ waves have been observed in cell cultures (Harris-White et al., 1998) with speeds that vary from 10 to $30 \mu \mathrm{m} / \mathrm{s}$ (Charles et al., 1992; Innocenti et al., 2000; Newman and Zahs, 1997; Yagodin et al., 1995; 1994) and range of propagation in the order of $200 \mu \mathrm{m}$ (Guthrie et al., 1999).

Two mechanisms for intercellular astrocytic $\mathrm{Ca}^{2+}$ signaling have been suggested in the literature. Initially, $\mathrm{Ca}^{2+}$ waves were thought to spread as a result of gap-junctionmediated metabolic coupling between astrocytes (Charles et al., 1992; Sanderson et al., 1994; Sneyd et al., 1995). According to this hypothesis, $\mathrm{IP}_{3}$ diffuses between astrocytes through gap junctions, stimulates the release of $\mathrm{Ca}^{2+}$ from intracellular ER stores of neighboring astrocytes and consequently leads to a calcium wave. There exists experimental evidence in support of this hypothesis. First, C6 glioma cells, which express few gap junctions do not exhibit $\mathrm{Ca}^{2+}$ waves unless connexins are artificially expressed (Charles et al., 1992). Moreover, caged $\mathrm{IP}_{3}$ experiments have indicated that $\mathrm{IP}_{3}$ diffuses through gap junctions and photolysis experiments have shown $\mathrm{Ca}^{2+}$ waves propagating in non-injected cells adjacent to those that were injected with the caged compound (Leybaert et al., 1998). However, the intercellular $\mathrm{Ca}^{2+}$ waves observed in these experiments usually propagated for much shorter distances (2-3 cell diameters) than those observed in cultures. Thus, the Achille's heel of the $\mathrm{IP}_{3}$-mediated hypothesis (intercellular mode of communication) is that $\mathrm{IP}_{3}$ diffusion by itself does not seem sufficient to account for the range of the wave 
propagation. As supported by mathematical studies (Sneyd et al., 1994; Sneyd et al., 1995), there should be a form of regeneration.

Such a regeneration mechanism is present in the second hypothesis that has been suggested to explain the experimentally observed range of $\mathrm{Ca}^{2+}$ wave propagation. This hypothesis involves an extracellular agonist (extracellular mode of communication) and was basically supported by experiments showing that a wave of elevated intracellular $\mathrm{Ca}^{2+}$ wave can "jump" the gap between two groups of cultured astrocytes separated by cell-free lanes. The $\mathrm{Ca}^{2+}$ can pass between disconnected cells as long as the gap is less than $\sim 120 \mu \mathrm{m}$ (Hassinger et al., 1995). It has also been shown that $\mathrm{Ca}^{2+}$ waves are blocked by purinergic receptor antagonists and by pretreatment with apyrase, an ATP degrading enzyme (Guthrie et al., 1999). Moreover, local stimulation with ATP has been found to be sufficient to evoke the experimentally observed $\mathrm{Ca}^{2+}$ waves (Guthrie et al., 1999). These experiments provided evidence that the aforementioned extracellular mode of astrocytic $\mathrm{Ca}^{2+}$ signaling is mediated by ATP released by stimulated astrocytes (Guthrie et al., 1999).

Newman has recently shown that the ATP-mediated extracellular pathway for $\mathrm{Ca}^{2+}$ wave propagation is also used by retinal glial cells. He utilized a bioluminescence assay to show that in addition to intercellular $\mathrm{Ca}^{2+}$ waves, ATP released by local mechanical stimulation of astrocytes also spreads in the form of a wave in retinal glial cells (Newman, 2001). The peak ATP concentration was approximately $78 \mu \mathrm{M}$, while the range of propagation greater than $100 \mu \mathrm{m}$. Moreover, the ATP wave was found to be faster than the corresponding $\mathrm{Ca}^{2+}$ wave, and the range of the $\mathrm{Ca}^{2+}$ wave was found to be smaller than that of the corresponding ATP wave. Finally, Innocenti et al. (2000) have shown that glutamate released from stimulated cultured astrocytes also 
propagates in the form of a wave, at an average speed of $26 \mu \mathrm{m} / \mathrm{s}$ and extending to $\sim 100 \mu \mathrm{m}$.

Recent reports have shed light into the physiological significance of astrocyte wave propagation by providing evidence for the dynamic interaction between astrocytes and neurons. Nedergaard (1994) showed that focal electrical stimulation of single astrocytes in mixed cultures of rat forebrain astrocytes and neurons triggers a wave of calcium increase, propagating from astrocyte to astrocyte, while neurons resting on these astrocytes respond with large increases in their cytosolic $\mathrm{Ca}^{2+}$ concentration. Kang et al. (1998) demonstrated that interneuronal firing elicits transient elevations of intracellular $\mathrm{Ca}^{2+}$ concentration in neighboring astrocytes, which, upon stimulation, potentiate miniature inhibitory postsynaptic currents (mIPSCs) in pyramidal neurons. Newman and Zahs showed that spike activity of neurons within the ganglion cell layer changed, when $\mathrm{Ca}^{2+}$ waves in astrocytes and Müller cells reached these neurons (Newman and Zahs, 1997).

As indicated by many of the aforementioned experimental studies, ATP plays a key role in intracellular signaling as well as wave propagation in astrocytic cellular networks. Despite recent progress, the mechanism by which ATP is released after stimulation is still largely unknown. Two main hypotheses have been postulated in the literature. The first hypothesis accounts for $\mathrm{Ca}^{2+}$-dependent ATP release (Cotrina et al., 1998) and the second for $\mathrm{IP}_{3}$-dependent ATP release (Wang et al., 2000). The focus of this work is the investigation of the consequences of each hypothesis on the behavior at the single-cell level dynamics and the resulting wave propagation characteristics in entire cell cultures.

Due to the complexity and the partial knowledge of the underlying biological mechanisms, understanding what controls wave propagation and blocking is a 
complicated task. Mathematical modeling and computations can obviously help in examining closely the consequences of different hypotheses about the key signaling pathways. To this end, in the following section we develop a model that describes signal transduction and wave propagation in astrocytic cellular networks. The model consists of a subsystem that describes $\mathrm{Ca}^{2+}$ release from internal stores and equations which model the $\mathrm{IP}_{3}$ production and ATP release, according to the aforementioned biological hypotheses. Using tools from bifurcation theory, we investigate the consequences of each biological hypothesis and we study the effects of key parameters on the transient and asymptotic behavior at the single-cell level. To study coupled astrocytic networks, we incorporate the model in a reaction diffusion framework. We assume that astrocyte coupling occurs in a homogeneous cell culture through the diffusion of extracellular ATP and we study the relationship between different single-cell ATP release mechanisms and wave generation, propagation and blocking characteristics. Due to the homogeneity assumption, we only consider focal stimulation of cell cultures, since simulations of bath application of ATP would predict the same results at the distributed level as those predicted at the single-cell level in a spatially homogeneous domain.

\section{MODEL}

We start our modeling studies by describing the mechanisms responsible for the release of $\mathrm{Ca}^{2+}$ into the cytosol. After obtaining quantitative insight into the behavior of this calcium subsystem, we expand this single-cell model to include ATP and $\mathrm{IP}_{3}$ dynamics. Finally, we incorporate the entire model in a reaction-diffusion framework that allows us to study the relationship between wave propagation/blocking phenomena and single-cell mechanisms. 


\section{Calcium subsystem}

$\mathrm{Ca}^{2+}$ dynamics have been studied extensively over the past two decades and several models have been presented in the literature. In our study, we have chosen to work with the Li-Rinzel (Li and Rinzel, 1994) reduced version of the De YoungKeizer model (De Young and Keizer, 1992) in order to capture the dynamics of the $\mathrm{Ca}^{2+}$ subsystem, because this model strikes the best balance between generality and simplicity. The Li-Rinzel model consists of equations 1 to 7 . Eq. 1 is a mass balance for $\mathrm{Ca}^{2+}$ in which the following fluxes are considered: efflux from the ER through $\mathrm{IP}_{3} \mathrm{R}$ channels as well as $\mathrm{Ca}^{2+}$ leakage and influx through an ATP-ase pump. Eq. 2 is derived from the scheme proposed by De Young and Keizer for the kinetics of the $\mathrm{IP}_{3} \mathrm{R}$ by using singular perturbation arguments ( $\mathrm{Li}$ and Rinzel, 1994). In this equation, $\mathrm{h}$ is a dimensionless lumped variable that contains information about the fraction of open channels.

$$
\begin{aligned}
& \frac{\mathrm{dC}}{\mathrm{dt}}=-\left(\mathrm{c}_{1} \cdot \mathrm{v}_{1} \cdot \mathrm{m}_{\infty}{ }^{3} \cdot \mathrm{h}^{3}+\mathrm{c}_{1} \cdot \mathrm{v}_{2}\right) \cdot\left(\mathrm{C}-\left[\mathrm{Ca}_{\mathrm{er}}^{2+}\right]\right)-\frac{\mathrm{v}_{3} \cdot \mathrm{C}^{2}}{\kappa_{3}{ }^{2}+\mathrm{C}^{2}} \\
& \frac{\mathrm{dh}}{\mathrm{dt}}=\frac{\mathrm{h}_{\infty}-\mathrm{h}}{\tau_{\mathrm{h}}}
\end{aligned}
$$

I and $\mathrm{C}$ denote intracellular $\mathrm{IP}_{3}$ and $\mathrm{Ca}^{2+}$ concentrations, respectively and:

$$
\begin{aligned}
& \mathrm{m}_{\infty}=\left(\frac{\mathrm{I}}{\mathrm{I}+\mathrm{d}_{1}}\right) \cdot\left(\frac{\mathrm{C}}{\mathrm{C}+\mathrm{d}_{5}}\right) \\
& \tau_{\mathrm{h}}=\frac{1}{\mathrm{a}_{2} \cdot\left(\mathrm{Q}_{2}+\mathrm{C}\right)} \\
& \mathrm{h}_{\infty}=\frac{\mathrm{Q}_{2}}{\mathrm{Q}_{2}+\mathrm{C}}
\end{aligned}
$$




$$
\begin{aligned}
& {\left[\mathrm{Ca}_{\mathrm{er}}^{2+}\right]=\frac{\mathrm{c}_{0}-\mathrm{C}}{\mathrm{c}_{1}}} \\
& \mathrm{Q}_{2}=\mathrm{d}_{2} \frac{\mathrm{I}+\mathrm{d}_{1}}{\mathrm{I}+\mathrm{d}_{3}}
\end{aligned}
$$

The Li and Rinzel model does not exhibit excitable behavior if the original parameter set (used by De Young, Keizer and Li, Rinzel) is employed (for definition and conditions of excitability see (FitzHugh, 1960; Murray, 1989)). However, it has been experimentally shown that $\mathrm{Ca}^{2+}$ dynamics are excitable in a variety of cells, including astrocytes (Bootman et al., 1992; Charles, 1998; Lechleiter et al., 1991; Neylon and Irvine, 1990; Putney, 1993; Shao and McCarthy, 1995; Woods et al., 1987). Detailed investigation of the Li-Rinzel model using phase plane techniques has shown that there exists a clear separation of the timescales of $\mathrm{C}$ and $\mathrm{h}$. Moreover two parameters were found able to significantly affect excitability by making the $\mathrm{Ca}^{2+}$ nullcline sufficiently cubic-shaped: a) The dissociation constant for $\mathrm{Ca}^{2+}$ binding to activating site (parameter $d_{5}$ ), which influences the extent to which the Calcium Induced Calcium Release (CICR (Roderick et al., 2003)) mechanism acts. Increase of this parameter promotes excitability, as it makes CICR more pronounced. b) The threshold constant that affects the operation of the pump (parameter $\kappa_{3}$ ). Lower values activate the pump at lower $\mathrm{Ca}^{2+}$ concentrations, thus promoting all-or-nothing type excitable responses. Since the original parameter values for the channel kinetics (including $\mathrm{d}_{5}$ ), suggested by De Young and Keizer, were shown to yield excellent agreement with experimental data on the fraction of open channels with respect to $\mathrm{IP}_{3}$ concentration (Bezprozvanny et al., 1991), we chose to change the value of parameter $\kappa_{3}$ in order to obtain excitable responses. Fig. 2 shows the nullclines, the vector field and the corresponding transient dynamics for the two cases of the original and 
modified values of the pump threshold constant. As shown in the figure, in the case where $\kappa_{3}$ is half that of the original value the cubic shape of the nullcline is sufficiently pronounced to evoke excitable behavior.

Furthermore, in Fig. $3 a, b$ the bifurcation diagrams with respect to the $\mathrm{IP}_{3}$ concentration for the two values of $\kappa_{3}$ are shown and in Fig. $3 c, d$ the periods of the oscillations are plotted. We note that these and all bifurcation diagrams were computed with XppAut (Ermentrout, 2002); solid lines represent stable steady states and dashed lines unstable ones, while filled circles denote stable periodic orbits and open circles unstable ones; for limit cycles the lower and upper points of the oscillation are plotted so that the amplitude can be illustrated. First, notice that $\mathrm{Ca}^{2+}$ concentrations lie in a range of $0.01-1.2 \mu \mathrm{M}$. This compares well with experimental data, as the resting $\mathrm{Ca}^{2+}$ concentrations in astrocytes have been found to be around 0.1 $\mu \mathrm{M}$ and raise up to $0.8 \mu \mathrm{M}$ after stimulation (Charles, 1998; Charles et al., 1991). Moreover, notice that for the original parameter set the amplitude of the stable oscillation changes noticeably with the $\mathrm{IP}_{3}$ concentration while the period changes less than $5 \mathrm{~s}$. However, for the parameter set that exhibits excitable behavior, the amplitude of the oscillations remains nearly constant, whereas the period changes orders of magnitude, due to an infinite period bifurcation at the knee around $\left[\mathrm{IP}_{3}\right]=$ $0.5 \mu \mathrm{M}$. These oscillations are thus shown to be frequency encoded, a phenomenon closely related to excitability (Mishchenko and Rozov, 1980; Tang and Othmer, 1995).

\section{IP $_{3}$ and ATP dynamics}

The model for calcium dynamics assumes that $\mathrm{IP}_{3}$ remains at constant concentrations in the cytosol. However, it is known that $\mathrm{IP}_{3}$ is produced when 
astrocytes are stimulated with a neurotransmitter, such as ATP (Kastritsis et al., 1992; Wang et al., 2000). As shown in experimental data by Kastritsis et al. (1992), ATPmediated $\mathrm{IP}_{3}$ production follows Michaelis Menten kinetics. Therefore, in order to account for $\mathrm{IP}_{3}$ dynamics we write a mass balance for intracellular $\mathrm{IP}_{3}$. with a Michaelis Menten production term, in which positive $\mathrm{Ca}^{2+}$ feedback is taken into account in the way suggested in (De Young and Keizer, 1992); a dimensionless parameter $\left(\alpha_{5}\right)$ quantifies the extent of the feedback. Degradation of $\mathrm{IP}_{3}$ is assumed to be linear.

$$
\frac{\mathrm{dI}}{\mathrm{dt}}=\mathrm{v}_{4} \cdot \frac{\mathrm{H}}{\kappa_{4}+\mathrm{H}} \cdot \frac{\mathrm{C}+\left(1-\alpha_{5}\right) \cdot \kappa_{5}}{\mathrm{C}+\kappa_{5}}-\mathrm{v}_{6} \cdot \mathrm{I}
$$

Clearly, the dynamics of $\mathrm{IP}_{3}$ are coupled with those of extracellular ATP $(\mathrm{H})$. As described in the introduction, ATP stimulates the cell, but the cell also releases ATP into the extracellular space through an unknown mechanism which we intend to investigate. To this end, ATP release is assumed to be either $\mathrm{Ca}^{2+}$-dependent or $\mathrm{IP}_{3}{ }^{-}$ dependent. This is modeled by utilizing a generic release term $c_{7} \cdot v_{7} \cdot F(C) \cdot G(I)$. Since one of the objectives of this paper is to investigate the potential, unknown role of $\mathrm{Ca}^{2+}$ and/or $\mathrm{IP}_{3}$ in ATP release, we considered quite general expressions for $\mathrm{F}(\mathrm{C})$ and $\mathrm{G}(\mathrm{I})$ that can describe both activating and/or inhibitory roles of these signals depending on their intracellular levels. To distinguish between the two mechanisms, in the case of $\mathrm{IP}_{3}$-dependent ATP release, function $\mathrm{F}(\mathrm{C})$ is set equal to 1 , while $\mathrm{G}(\mathrm{I})$ has a bell-shaped functional form to account for the possible promoting or inhibitory role of $\mathrm{IP}_{3}$ in ATP release for different $\mathrm{IP}_{3}$ concentrations. Similarly, in the case of $\mathrm{Ca}^{2+}$-dependent ATP release, $\mathrm{G}(\mathrm{I})$ is set equal to 1 and $\mathrm{F}(\mathrm{C})$ takes the form of a bellshaped function to account for concentration-dependent, promoting and/or inhibitory effects of $\mathrm{Ca}^{2+}$ on ATP release. The functional forms for $\mathrm{F}(\mathrm{C})$ and $\mathrm{G}(\mathrm{I})$ have been 
constructed such that $\mathrm{F}(\mathrm{C})(\mathrm{G}(\mathrm{I}))$ reaches a maximum when $\mathrm{C}=\mathrm{C}_{\max }\left(\mathrm{I}=\mathrm{I}_{\max }\right)$, and is equal to $\mathrm{F}_{\mathrm{o}}\left(\mathrm{G}_{\mathrm{o}}\right)$ for $\mathrm{C}=0 \mu \mathrm{M}(\mathrm{I}=0 \mu \mathrm{M})$, where ATP is released only due to leakage. We considered a small, non-zero value for $F_{o}$ and $G_{0}$ to describe basal ATP release when $\mathrm{C}=0 \mu \mathrm{M}(\mathrm{I}=0 \mu \mathrm{M})$. Also, the function is normalized, which means that the maximum value is equal to unity. ATP degradation to adenosine is assumed to follow Michaelis-Menten kinetics. Therefore the mass balance is:

$$
\frac{\mathrm{dH}}{\mathrm{dt}}=\mathrm{c}_{7} \cdot \mathrm{v}_{7} \cdot \mathrm{F}(\mathrm{C}) \cdot \mathrm{G}(\mathrm{I})-\mathrm{v}_{8} \cdot \frac{\mathrm{H}}{\kappa_{8}+\mathrm{H}}
$$

where:

$$
\begin{aligned}
& F(C)=\left\{\begin{array}{l}
\frac{\frac{\mathrm{F}_{0}}{\mathrm{~F}_{0}-1}-2 \cdot \frac{\mathrm{C}}{\mathrm{C}_{\max }}}{\frac{1}{\mathrm{~F}_{0}-1}-\left(\frac{\mathrm{C}}{\mathrm{C}_{\max }}\right)^{2}} \\
1 \text { for } \mathrm{IP}_{3}-\text { dependent ATP release } \mathrm{Ca}^{2+}-\text { dependent ATP release }
\end{array}\right. \\
& G(I)=\left\{\begin{array}{l}
1 \text { for } \mathrm{Ca}^{2+}-\text { dependent ATP release } \\
\frac{\mathrm{G}_{0}-2 \cdot \frac{\mathrm{I}}{\mathrm{G}_{0}-1}}{\frac{1}{\mathrm{G}_{0}-1}-\left(\frac{\mathrm{I}}{\mathrm{I}_{\max }}\right)^{2}} \quad \text { for } \mathrm{IP}_{3}-\text { dependent ATP release }
\end{array}\right.
\end{aligned}
$$

Thus, the full single-cell model describes the $\mathrm{Ca}^{2+}$ mobilization from the ER and the release of ATP to the extracellular space when an astrocyte is stimulated by an increase in the extracellular ATP concentration. It consists of Eq. 1-11 with parameter values shown in Tables 1-3.

\section{Distributed model}

In order to study wave propagation in a coupled network of astrocytes, we 
incorporated these four equations into a reaction-diffusion framework with no flux boundary conditions. The general formulation is as follows:

$$
\begin{array}{ll}
\frac{\partial \underset{\sim}{\mathrm{u}}}{\partial \mathrm{t}}=\underset{\sim}{\mathrm{D}} \cdot \nabla_{\sim}^{2} \underset{\sim}{\mathrm{u}}+\underset{\sim}{\mathrm{f}}(\underset{\sim}{\mathrm{u}}) & (\mathrm{x}, \mathrm{y}) \in \mathrm{D} \\
\underset{\sim}{\mathrm{u}}(\mathrm{x}, \mathrm{y}, 0)={\underset{\sim}{\mathrm{u}}}_{0}(\mathrm{x}, \mathrm{y}) & \left.\underset{\sim}{\mathrm{n}} \cdot \nabla_{\sim}^{\mathrm{u}}\right|_{\partial \mathrm{D}}=0
\end{array}
$$

where:

$$
\underset{\sim}{\mathrm{u}}=\left[\begin{array}{c}
{[\mathrm{ATP}]} \\
{\left[\mathrm{IP}_{3}\right]} \\
{\left[\mathrm{Ca}^{2+}\right]} \\
\mathrm{h}
\end{array}\right], \underset{\sim}{\mathrm{D}}=\left[\begin{array}{cccc}
\mathrm{D}_{\mathrm{ATP}} & 0 & 0 & 0 \\
0 & \mathrm{D}_{\mathrm{IP}_{3}} & 0 & 0 \\
0 & 0 & 0 & 0 \\
0 & 0 & 0 & 0
\end{array}\right], \underset{\sim}{\mathrm{f}}(\underset{\sim}{\mathrm{u}})=\left[\begin{array}{c}
\mathrm{f}_{\mathrm{ATP}} \\
\mathrm{f}_{\mathrm{IP}_{3}} \\
\mathrm{f}_{\mathrm{Ca}^{2+}} \\
\mathrm{f}_{\mathrm{h}}
\end{array}\right]
$$

The reaction terms are the ones that appear as right hand sides in Eq. 1, 2, 8 and 9. Notice that in order to accommodate the intercellular and extracellular modes of astrocytic communication, we allow for diffusion of ATP and/or $\mathrm{IP}_{3}$ although our focus is the former. $\mathrm{Ca}^{2+}$ diffusion has been experimentally found to have negligible contribution to astrocytic wave propagation due to buffering effects (Allbritton et al., 1992), therefore it was omitted. Clearly, $D_{h}=0 \mu \mathrm{m}^{2} / \mathrm{s}$, since channels do not diffuse. This reaction-diffusion model was simulated in both one and two dimensional domains, with appropriate ATP focal stimuli as initial conditions. Simulations and results will be described in the following section.

To solve the reaction-diffusion problem numerically, a variety of Finite Difference and Finite Element schemes for spatial discretization were implemented, in conjunction with different time integrators. To verify the validity of these algorithms, we applied them to model reaction-diffusion problems where analytical solutions can be easily obtained. Moreover, through comparison with results published in the literature, we also saw that our numerical schemes can successfully simulate the 
reaction-diffusion problem with FitzHugh-Nagumo reaction terms. For the onedimensional case, a Central Difference spatial discretization scheme in conjunction with the Trapezoid Rule (Adams-Moulton $2^{\text {nd }}$ order) for time integration was found to offer the best results. In two-dimensional simulations, a nine-point Finite Difference approximation of the Laplacian with Runge-Kutta $4^{\text {th }}$ order as the time integrator was shown to be the most computationally efficient.

\section{RESULTS}

In this section, we will present results from our simulation studies, aiming at quantitatively elucidating the relationship between single-cell signal transduction mechanisms and wave propagation properties in a coupled astrocytic network. Specifically, we will investigate the qualitative and quantitative differences observed at both the single-cell and cell network levels due to $\mathrm{Ca}^{2+}$ - or $\mathrm{IP}_{3}$-dependent ATP release mechanisms.

\section{$\mathrm{Ca}^{2+}$-dependent ATP release}

The overall system at the single-cell level can be thought as an assembly of two coupled modules: the driver ATP-IP 3 system and the driven $\mathrm{Ca}^{2+}$ subsystem. In the case of $\mathrm{Ca}^{2+}$-dependent ATP release, the "communication" or coupling between these two, occurs in terms of two signals: the $\mathrm{IP}_{3}$ concentration, which is the output of the driver system and the input to the driven, and the $\mathrm{Ca}^{2+}$ concentration, which is the output of the driven system and the input to the driver system (see Fig. 4 for a schematic of the interactions). As mentioned earlier, the $\mathrm{Ca}^{2+}$ concentrations lie in a range of $0.01-1.2 \mu \mathrm{M}$. Therefore, if we set the maximum of $\mathrm{F}(\mathrm{C})$ at $1.5 \mu \mathrm{M}$, we essentially assume that the driven $\mathrm{Ca}^{2+}$ subsystem mainly exerts positive feedback on ATP release, while for lower values of $\mathrm{C}_{\max }$, some negative feedback is also exerted 
for higher $\mathrm{Ca}^{2+}$ concentrations.

Fig. 5 shows the local bifurcation diagram for the four variables of the system in the case where $\mathrm{Ca}^{2+}$ plays an activating role in the release of ATP $\left(\mathrm{C}_{\max }=1.5 \mu \mathrm{M}\right)$. The main bifurcation parameter is the inverse of the $\mathrm{IP}_{3}$ degradation rate. This choice is due to the availability of a range of experimentally measured values of this parameter (degradation rate constants from 0.0 to $0.284 \mathrm{~s}^{-1}$ in mouse neuroblastoma cells (Wang et al., 1995), yet, to our knowledge, no such studies have been performed on astrocytes).

First, notice that lower $\mathrm{IP}_{3}$ degradation rates lead to higher equilibrium ATP concentrations. This is a direct consequence of the fact that slower $\mathrm{IP}_{3}$ degradation increases the $\mathrm{IP}_{3}$ equilibrium concentration, as can be easily seen from Eq. 8. This, in turn, leads to an increase in the equilibrium $\mathrm{Ca}^{2+}$ concentration as also shown in Fig. 5 c. However, for values of $\mathrm{Ca}^{2+}$ concentration close to $\mathrm{C}_{\max }, \mathrm{Ca}^{2+}$ enhances ATP release, thus leading to the observed increase in the equilibrium ATP concentration. Furthermore, notice that the $\mathrm{ATP}$ and $\mathrm{IP}_{3}$ bifurcation structures, strikingly resemble that of the $\mathrm{Ca}^{2+}$ subsystem. A stable sigmoid branch of steady states becomes unstable at a subcritical Hopf bifurcation which occurs before the first turning point bifurcation. It becomes stable again through another subcritical Hopf which occurs after the second turning point. The observed branch of periodic orbits is generated through an infinite period bifurcation that occurs right before the knee. The periodic orbits become unstable through a turning point bifurcation and they vanish at the second subcritical Hopf. Moreover, the amplitude of the stable periodic orbits is almost constant for all species under consideration, while the period varies significantly (results not shown). Thus, for a significant region of the parameter space, the entire system exhibits frequency-encoded oscillations. In addition, for values of 
$1 / \mathrm{v}_{6}$ lower than the turning point bifurcation value, the entire system is excitable. Specifically, the ATP stimulus leads to a transient elevation of intracellular $\mathrm{IP}_{3}$ concentration. When the $\mathrm{IP}_{3}$ concentration becomes sufficiently large, it shifts the $\mathrm{Ca}^{2+}$ dynamics into the oscillatory regime, thus a $\mathrm{Ca}^{2+}$ spike is produced. This leads to ATP release into the extracellular space and subsequently $\mathrm{IP}_{3}$ is regenerated. However, if the $\mathrm{IP}_{3}$ degradation dominates over the production, no further $\mathrm{Ca}^{2+}$ spikes are observed, because the $\mathrm{IP}_{3}$ concentration drops below the window that forces $\mathrm{Ca}^{2+}$ to oscillate. Thus, only excitable behavior is observed (values of $1 / \mathrm{v}_{6}$ before the knee). On the other hand, if the $\mathrm{IP}_{3}$ degradation is relatively small compared to $\mathrm{IP}_{3}$ production, persistent oscillations are observed (values of $1 / \mathrm{v}_{6}$ in the oscillatory regime).

The predicted behavior can be explained as follows. By analyzing the ATP-IP 3 system as a module independent of $\mathrm{Ca}^{2+}$ concentration (the latter was treated as a parameter), it was shown that the former does not posses excitability and frequency encoding properties on its own (see Appendix for a proof). Thus, the behavior of the ATP and $\mathrm{IP}_{3}$ concentrations depicted in Fig. 5 is due to the fact that the excitable $\mathrm{Ca}^{2+}$ subsystem exerts a purely positive feedback on the driver ATP-IP ${ }_{3}$ system. Hence, the ATP and $\mathrm{IP}_{3}$ bifurcation structures are essentially "inherited" from the $\mathrm{Ca}^{2+}$ subsystem, which is excitable and exhibits frequency encoded oscillations by itself as already shown in Fig. 3.

A drastically different behavior of the system is observed if ATP release is significantly inhibited by high $\mathrm{Ca}^{2+}$ concentrations (this happens for lower values of $\left.\mathrm{C}_{\max }\right)$. Fig. $6 a$ shows the ATP bifurcation diagram for a different extent of $\mathrm{Ca}^{2+}$ inhibition $\left(\mathrm{C}_{\max }=0.5 \mu \mathrm{M}\right)$. First, notice that the steady state now appears in higher concentrations, because $\mathrm{C}_{\max }$ can be reached and the maximum possible positive 
feedback in ATP can be attained. After that, the steady state falls to lower values because of the inhibitory effects. For even stronger inhibition effects $\left(\mathrm{C}_{\max }=0.1 \mu \mathrm{M}\right)$, a rather interesting folding of the steady state branch appears (Fig. $6 b$ ). The cross point of the folding is not a singular point as it may seem at first glance; it appears this way because of the projection of the 4-dimensional bifurcation structure on a 2dimensional plane. Fig. 7 explains how strong inhibition creates the folding. The bottom sketch (Fig. 7 e) presents the $\mathrm{Ca}^{2+}$ bifurcation structure. We note the region of $\mathrm{Ca}^{2+}$ concentration where $\mathrm{Ca}^{2+}$ inhibits ATP release for the chosen value of $\mathrm{C}_{\max }$, as well as the region where $\mathrm{Ca}^{2+}$ enhances ATP release. The left upper sketch (Fig. 7 a) is the ATP bifurcation diagram, drawn as if no inhibition took place. Due to the inhibition at values higher than $\mathrm{C}_{\max }$ the branch above the dashed curve folds and creates the characteristic "lasso" shown in Fig. 7 b. A major effect of this increasingly inhibitory role of $\mathrm{Ca}^{2+}$ in ATP release (represented by the lower $\mathrm{C}_{\max }$ values) is that the overall excitability of the system is suppressed. Thus, despite the fact that the underlying $\mathrm{Ca}^{2+}$ subsystem remains excitable, the excitable behavior is no longer inherited to the ATP-IP 3 system. Therefore, a purely inhibitory role of $\mathrm{Ca}^{2+}$ on ATP release leads to non-excitable single-astrocyte behavior.

We further explored the effect of the ATP degradation Michaelis-Menten constant $\left(\kappa_{8}\right)$, since this parameter is known to vary greatly not only between cells of different types, but also within the same cell population (Cunha et al., 1998; Dunwiddie et al., 1997). Lower values of $\kappa_{8}$ result in lower ATP steady state concentrations for the same value of $\mathrm{IP}_{3}$ degradation rate. As a result, $\mathrm{IP}_{3}$ production is reduced, and the $\mathrm{Ca}^{2+}$ subsystem is forced out of the oscillatory regime. Thus, the beginning of the oscillatory regime will move to the right. This is shown in Fig. 8 in which the point where the oscillatory regime starts is plotted as a function of $\kappa_{8}$. The increase of the 
$\mathrm{IP}_{3}$ production Michaelis-Menten constant $\left(\kappa_{4}\right)$ has a very similar effect on the system (results not shown).

The extracellular volume can be experimentally manipulated in astrocytic cell cultures. The effect of such a manipulation can be quantitatively studied with our model by changing parameter $\mathrm{c}_{7}$, which is the ratio of the cytosolic volume over the extracellular volume. Small values of $c_{7}$ indicate large extracellular volumes. The bifurcation diagram (Fig. 9) reveals two interesting effects of the increase of the extracellular volume (decrease of $c_{7}$ ). First, the steady state of ATP is lowered significantly, as the production term for ATP at the extracellular space is lowered. Second, stable periodic solutions cease to exist, due to the domination of ATP degradation over ATP production. Furthermore, transient simulations reveal that excitability of the $\mathrm{Ca}^{2+}$ system is diminished by dilution, because no significant amount of $\mathrm{IP}_{3}$ is produced in order to force the $\mathrm{Ca}^{2+}$ subsystem into the oscillatory regime. Similar is the effect of parameter $\mathrm{v}_{7}$, which is the maximum ATP release rate. The effect of the maximum ATP degradation rate $\left(\mathrm{v}_{8}\right)$ is the opposite of that of $\mathrm{v}_{7}$ : domination of the degradation term (large values of $\mathrm{v}_{8}$ ) lowers the ATP steady state and suppresses oscillations.

Finally, the effect of $\mathrm{Ca}^{2+}$ feedback in $\mathrm{IP}_{3}$ production was investigated, as there exists experimental evidence indicating that $\mathrm{Ca}^{2+}$ is responsible for PLC activation (Mouillac et al., 1990; Smrcka et al., 1991). To study this effect, the value of parameter $\mathrm{a}_{5}$ is modified. For $\mathrm{a}_{5}=1, \mathrm{Ca}^{2+}$ exerts feedback at the fullest possible extent, thus no production of $\mathrm{IP}_{3}$ occurs if no $\mathrm{Ca}^{2+}$ is present in the cytosol. For values of $\mathrm{a}_{5}$ less than unity and greater than zero, $\mathrm{Ca}^{2+}$ exerts only partial feedback, but there is also "leak" $\mathrm{IP}_{3}$ production not attributed to $\mathrm{Ca}^{2+}$. The bifurcation diagrams in Fig. 10 show that for stronger positive feedback (higher value of $a_{5}$ ), stable oscillations 
become suppressed. In this case, when $\mathrm{Ca}^{2+}$ exists in low or moderate concentrations, it hinders the production of $\mathrm{IP}_{3}$ and consequently its own release from the ER. Thus, the positive and negative feedback loop that is necessary for the oscillations cannot manifest itself. Instead of stable oscillations, only a branch of unstable oscillations exists, which is destroyed through an infinite period bifurcation.

After obtaining quantitative insights into the behavior of single cells, we focus on investigating how single-cell mechanisms modulate cell culture behavior. We first consider a one-dimensional coupled astrocytic network (i.e. a line series of cells). We assume medium homogeneity in the sense that production/degradation or release/uptake of species as well as diffusion occurs throughout the simulated domain. In a real cell culture however, there exist inhomogeneities such as reaction free zones, where only diffusion takes place (gap junctions). Also, the two modes of communication occur in different control volumes, which is a source of inhomogeneity itself. We did not investigate such geometric effects here, since our intention was to focus on the relationship between single-cell signal transduction mechanisms and wave propagation properties.

In all distributed simulations presented, the extracellular pathway is assumed to be dominant (thus only extracellular ATP is allowed to diffuse). However, we have performed simulations in which both pathways are present (both extracellular ATP and intracellular $\mathrm{IP}_{3}$ are allowed to diffuse) or only the intracellular pathway is dominant (only $\mathrm{IP}_{3}$ diffuses). The results of such simulations are qualitatively the same. This happens because the dynamics of $\mathrm{IP}_{3}$ production are strictly dependent on ATP, which receives feedback from $\mathrm{Ca}^{2+}$. Thus the two species that dominate in shaping the response of the system are $\mathrm{Ca}^{2+}$ and $\mathrm{ATP} ; \mathrm{IP}_{3}$ is just used as an intermediate messenger (see also supplemental text). Estimated values for ATP and 
$\mathrm{IP}_{3}$ diffusion coefficients were found in the literature: $\mathrm{D}_{\mathrm{ATP}}=330 \sim 500 \mu \mathrm{m}^{2} / \mathrm{s}$ at $20^{\circ} \mathrm{C}-37^{\circ} \mathrm{C}$ (Graaf et al., 2000; Newman, 2001) and $\mathrm{D}_{\mathrm{IP} 3}=283 \mu \mathrm{m}^{2} / \mathrm{s}$ (Allbritton et al., 1992).

Furthermore, the choice of parameter values for the distributed simulations has been made in such a way that the astrocytic network initially rests at a stable steady state, which gives basal concentrations for all the species. The reason is that we are interested in reproducing experimentally observed behaviors. In experiments with cultures or tissue slices of astrocytes, the cells are excited from a rest state. The concentrations of $\mathrm{Ca}^{2+}$ and ATP are within basal levels $\left(\left[\mathrm{Ca}^{2+}\right] \approx 0.1 \mu \mathrm{M}\right.$ and extracellular $[\mathrm{ATP}]<10 \mu \mathrm{M})$. Therefore the oscillatory regime is not of interest. Similarly, the regime in which a stable steady state with high concentrations exists (large $1 / \mathrm{v}_{6}$ values) is not of interest, since the concentrations of $\mathrm{Ca}^{2+}$ and/or ATP are well beyond the basal levels. However, these regimes, as well as the regime in which a stable steady state coexists with a stable limit cycle, were investigated and the simulations indicate that no chemical waves can be generated there (see supplemental text and figures). In the oscillatory regime, an ATP stimulus results in a shift of phase, which propagates by diffusional coupling to neighboring cells, yet this is not an chemical wave. In the regime where the upper steady state exists, an ATP stimulus merely produces a response since the cells are already excited and their $\mathrm{Ca}^{2+}$ levels are already high. This behavior is also observed in the regime in which the steady state coexists with the limit cycle.

The choice of a stimulation protocol was made on the basis of reproducing experimental results. To reproduce focal stimulation, in all 1-D simulations a narrow ATP square pulse in space was imposed, while cylindrical ATP pulses were used in 2D simulations. All cells were initially at rest. Focal stimulation initially raises the 
local levels of ATP, and consequently $\mathrm{IP}_{3}$ and $\mathrm{Ca}^{2+}$ only in a very small region of the astrocytic culture. Simulations of bath applications of agonist would not show any patterns that could explain experimental findings because we have assumed homogeneity of the astrocytic network. Thus, simulating the distributed network will yield the same results as if the single cell model were simulated. Therefore, we will only present results in which a focal ATP stimulus is applied to an astrocytic network which rests at the basal steady state.

Due to the excitable nature of the local dynamics, if $\mathrm{Ca}^{2+}$ has a sufficiently promoting role in ATP release (higher values of $\mathrm{C}_{\max }$ ), the system can generate and propagate waves. Fig. 11 shows such a traveling wave pulse generated by a focal ATP stimulus $\left(\mathrm{D}_{\mathrm{ATP}}=350 \mu \mathrm{m}^{2} / \mathrm{s}\right.$, no $\mathrm{IP}_{3}$ diffusion $)$. Due to the excitability properties of the entire system, in this case the wave has infinite range of propagation in both space and time. The wave speed was found to be proportional to the square root of the diffusion coefficient, in agreement with the theory of wave propagation. Since only one species diffuses, the diffusion coefficient just affects the space scaling (Fife, 1979). The observed wave speeds lie in a range of $10 \mu \mathrm{m} / \mathrm{s} \sim 20 \mu \mathrm{m} / \mathrm{s}$ which is in the same order of magnitude with experimentally observed wave speeds (Innocenti et al., 2000; Newman and Zahs, 1997).

We further expanded our analysis to simulate wave propagation in twodimensional homogeneous cultures. A particularly interesting pattern that has been observed experimentally is the formation of spiral waves (Harris-White et al., 1998; Jung et al., 1998). It has been suggested that spiral waves are the most stable type of waves in two-dimensional excitable media (Fernandez-Garcia et al., 1994) because their wavelength is less than of any other type of wave.

For generation of spiral waves we exploit a property termed "vulnerability", 
Specifically, a stimulus placed appropriately at an anisotropically excitable medium can generate a unidirectionally propagating wave (Starobin et al., 1994; Wiener and Rosenblueth, 1946). In two dimensions a sequence of two point ATP stimuli can generate ATP spirals as shown in Fig. 12. We note that the ATP spiral wave presented in Fig. 12 is accompanied by a similar $\mathrm{Ca}^{2+}$ spiral wave (results not shown). The first stimulus generates a circular wave that creates the desired anisotropy as it propagates: each cell undergoes a refractory period after excitation, but cells along the direction of propagation have been excited in different times. Cells close to the front were excited more recently so they are in the early refractory period, while the cells away form the front were excited earlier, so they are in the late refractory period. As a result, it is very difficult (if at all possible) to re-excite cells close to the front, but this doesn't apply to cells away from the front. Therefore, anisotropy arises. The second stimulus generates a circular front which breaks as it tries to propagate to cells that are in the early refractory period. We note that the stimulus must be carefully placed in space and time so that vulnerability is exhibited. Premature stimuli do not lead to wave generation at all and late stimuli generate circular fronts.

Spiral waves in the context of $\mathrm{Ca}^{2+}$ signaling have also been presented in previous modeling studies (Atri et al., 1993; Hofer et al., 2001; Wilkins and Sneyd, 1998). However, the authors considered the $\mathrm{IP}_{3}$-mediated intercellular mode of cellular coupling in conjunction with the existence of spatial inhomogeneities, such as gap junctions. Our results indicate that this mode of cellular signaling in the presence of reaction-free gap junctions is not the only mechanism that can explain spiral wave generation and propagation. In particular, ATP and $\mathrm{Ca}^{2+}$ spiral waves can be obtained if appropriate stimulation patterns are employed in homogeneous astrocytic cell cultures where the extracellular (ATP-mediated) mode of communication dominates 
astrocytic coupling.

\section{$\mathrm{IP}_{3}$-dependent ATP release}

As opposed to the bi-directional communication between the ATP-IP 3 and $\mathrm{Ca}^{2+}$ systems in the case of $\mathrm{Ca}^{2+}$-dependent ATP release, in this case, the two systems are coupled in a unidirectional fashion (see Fig. 13 for the interaction diagram). Specifically, the output of the ATP-IP $\mathrm{I}_{3}$ system ( $\mathrm{IP}_{3}$ concentration) drives the excitable $\mathrm{Ca}^{2+}$ subsystem, without $\mathrm{Ca}^{2+}$ feeding back to ATP. As a result, the local bifurcation structure of the ATP-IP 3 system is fundamentally different than in the case of $\mathrm{Ca}^{2+}$ dependent ATP release (compare Fig. 13 and 5). There exists a unique, globally stable ATP concentration for each value of the $\mathrm{IP}_{3}$ degradation rate, while oscillations and excitable behavior are no longer observed. A similar behavior is predicted for the $\mathrm{IP}_{3}$ concentration (not shown). In contrast, the $\mathrm{Ca}^{2+}$ system still exhibits frequency encoded oscillations and excitability. These bifurcation structures are a direct consequence of the lack of feedback from $\mathrm{Ca}^{2+}$ to the driver system, due to which the $\mathrm{Ca}^{2+}$ subsystem cannot "transmit" its special properties to the driver ATP-IP 3 system.

The range of the $\mathrm{IP}_{3}$ degradation rate $\left(\mathrm{v}_{6}\right)$ for which oscillatory behavior is observed in the $\mathrm{Ca}^{2+}$ subsystem, is a function of the extent of inhibition of $\mathrm{IP}_{3}$ in ATP release, as quantified by parameter $\mathrm{I}_{\max }$. The effect of $\mathrm{I}_{\max }$ is shown in Fig. 14 and can be understood in terms of the changes of the slope of the $\mathrm{IP}_{3}$ steady state. As discussed earlier, there is a specific $\mathrm{IP}_{3}$ concentration window leading to oscillatory $\mathrm{Ca}^{2+}$ dynamics (see Fig. 3). If the slope of the $\mathrm{IP}_{3}$ steady state concentration is high (first case, panels $a, b, \mathrm{I}_{\max }=1.5 \mu \mathrm{M}$ ), $\mathrm{IP}_{3}$ exits the regime for which $\mathrm{Ca}^{2+}$ oscillations are observed for higher $\mathrm{IP}_{3}$ degradation rates. As a result, the oscillatory regime will shrink, while, for smaller slopes (second case, panels $c, d, \mathrm{I}_{\max }=0.1 \mu \mathrm{M}$ ) the oscillatory regime is magnified. 
The sensitivity of these bifurcation structures to values of the parameters which quantify the effect of ATP degradation $\left(\kappa_{8}\right)$ and the feedback of ATP to $\operatorname{IP}_{3}\left(\kappa_{4}\right)$ was investigated. The analysis in the present situation is simplified in comparison to the $\mathrm{Ca}^{2+}$ dependent ATP release case: here the steady-state mass balances for $\mathrm{IP}_{3}$ and ATP (Eq. 8 and 9) can be solved separately as a $2 \times 2$ system. The $\mathrm{IP}_{3}$ concentration as a function of the main bifurcation parameter can be then used to predict the $\mathrm{Ca}^{2+}$ subsystem behavior. It was thus found that lower values for the ATP degradation Michaelis-Menten constant $\kappa_{8}$, result in lower ATP steady-state concentrations and consequently lower $\mathrm{IP}_{3}$ concentrations for the same $\mathrm{IP}_{3}$ degradation rate. This occurs because the ATP degradation term becomes dominant, consequently the $\mathrm{IP}_{3}$ produced is not enough to excite the $\mathrm{Ca}^{2+}$ subsystem. Therefore, stronger ATP degradation diminishes excitability; the same effect was observed for the $\mathrm{Ca}^{2+}$ dependent ATP release case. Furthermore, higher Michaelis-Menten constants for $\operatorname{IP}_{3}$ production $\left(\kappa_{4}\right)$ result in lower values for the $\mathrm{IP}_{3}$ production term for the same ATP concentration. Therefore the ATP steady-state concentrations shift to lower (higher) values in the region where $\mathrm{IP}_{3}$ has a promoting (inhibitory) effect. Moreover the peak ATP concentration as well as the oscillatory regime for the $\mathrm{Ca}^{2+}$ subsystem shift to lower $\mathrm{IP}_{3}$ degradation rates (higher $1 / \mathrm{v}_{6}$ values).

To study the qualitative differences between the two ATP release mechanisms with respect to their wave propagation characteristics, we performed detailed onedimensional simulations for the $\mathrm{IP}_{3}$-dependent ATP release case. As argued previously, we are interested in simulating a focal ATP stimulus applied to an astrocytic network which rests at the basal steady state. The other parameter regimes (oscillatory regime, upper steady state and regime of coexistence) are not of physiological interest and were found unable to support wave generation and 
propagation (see supplemental text).

Fig. 15 shows a representative example for the case where astrocytic communication occurs through the extracellular mode of communication $\left(\mathrm{D}_{\mathrm{ATP}}=350\right.$ $\mu \mathrm{m}^{2} / \mathrm{s}-\mathrm{D}_{\mathrm{IP} 3}=0 \mu \mathrm{m}^{2} / \mathrm{s}$ ). The stimulus is a square pulse (in space) superimposed on the basal ATP concentration. Notice the striking qualitative difference between this wave and the ones generated in the $\mathrm{Ca}^{2+}$-dependent ATP release case (Fig. 11). The wave here vanishes after propagating for several cell diameters.

The number of cells that the signal manages to excite depends on the amplitude of the stimulus. Moreover, for large enough stimuli ([ATP]>1mM) multiple $\mathrm{Ca}^{2+}$ waves with finite range of propagation are predicted (results not shown). These regeneration phenomena, which have also been experimentally observed (Salm and McCarthy, 1990), are a consequence of the prolonged high concentrations of $\mathrm{IP}_{3}$ produced locally as a result of high ATP concentrations, which keep the local $\mathrm{Ca}^{2+}$ dynamics in the oscillatory regime long enough for multiple spikes to be generated. However, we emphasize the fact that the pattern of this finite range of propagation is not influenced by the amplitude of the stimulus. It is also very robust to variations in the parameter values, such as the diffusion coefficient or parameters of the signal transduction pathway. The predicted pattern of finite range of propagation is a direct consequence of the single cell dynamics. In particular, the non-excitable ATP-IP 3 driver system (see Appendix A for a proof) drives the excitable $\mathrm{Ca}^{2+}$ subsystem. Due to the lack of $\mathrm{Ca}^{2+}$ feedback in ATP release in the case of $\mathrm{IP}_{3}$-dependent ATP release, the excitable properties of the $\mathrm{Ca}^{2+}$ subsystem cannot be transmitted to the driver ATP-IP 3 system. Hence, ATP regeneration is insufficient to sustain the wave and this explains the diffusion-like pattern for the propagation of the ATP signal that is shown in Fig. 15. Therefore, as soon as the extracellular ATP concentration, which drives $\mathrm{IP}_{3}$-mediated 
$\mathrm{Ca}^{2+}$ release, drops below a certain value, the $\mathrm{Ca}^{2+}$ subsystem will return to rest as well. As a result, despite the excitable properties of the $\mathrm{Ca}^{2+}$ subsystem, the generated ATP, $\mathrm{IP}_{3}$ and $\mathrm{Ca}^{2+}$ waves can only have a finite range of wave propagation. On the contrary in the $\mathrm{Ca}^{2+}$-dependent $\mathrm{ATP}$ release case, discussed earlier, $\mathrm{IP}_{3}$ is continuously regenerated due to the positive feedback that the excitable $\mathrm{Ca}^{2+}$ system exerts on ATP release. Thus, both the ATP and $\mathrm{Ca}^{2+}$ waves will never become blocked once generated in a spatially homogeneous medium.

We note that the aforementioned unidirectional coupling between $\mathrm{IP}_{3}$ and ATP constitutes a semi-regenerative mechanism for calcium wave propagation. A different mechanism has been postulated by Hofer et al. (2002) to explain such phenomena. Since this study focused on the intercellular pathway of astrocytic coupling, semiregeneration of calcium waves was explained on the basis of simultaneous intercellular diffusion of $\mathrm{IP}_{3}$ and $\mathrm{Ca}^{2+}$ through gap junctions. However, $\mathrm{Ca}^{2+}$ diffusion was found to have negligible contribution to astrocytic wave propagation due to buffering effects (Allbritton et al., 1992). Thus, the proposed mechanism of partial regeneration of $\mathrm{IP}_{3}$ and $\mathrm{Ca}^{2+}$ waves with finite range of propagation due to $\mathrm{IP}_{3}{ }^{-}$ dependent and not $\mathrm{Ca}^{2+}$-dependent ATP release offers an alternative explanation for the experimentally observed patterns.

In addition to the finite range of wave propagation, the aforementioned unidirectional coupling between the ATP-IP 3 and $\mathrm{Ca}^{2+}$ systems at the single-cell level can also explain several other experimental findings. In particular, it can explain the fact that the calcium wave does not propagate beyond the excursion range of the ATP wave (Wang et al., 2000) and that ATP waves travel faster than $\mathrm{Ca}^{2+}$ waves $(41 \mu \mathrm{m} / \mathrm{s}$ versus $28 \mu \mathrm{m} / \mathrm{s}$, data from (Newman, 2001)). Intracellular $\mathrm{Ca}^{2+}$ levels become elevated only in cells in which $\mathrm{IP}_{3}$ is above a certain threshold. Moreover, $\mathrm{IP}_{3}$ levels 
are modulated by ATP, which is not regenerated sufficiently due to the lack of $\mathrm{Ca}^{2+}$ feedback on ATP release. Thus, the range and speed of the $\mathrm{Ca}^{2+}$ wave can never exceed those of ATP and $\mathrm{IP}_{3}$. Moreover, Wang et al. (2000) observed a large decrease of the ATP signal as a function of space and time after the initial stimulation but only a modest attenuation of the $\mathrm{Ca}^{2+}$ signal. This cannot be explained if $\mathrm{Ca}^{2+}$ exerts a significant positive feedback on ATP release. However, it can be understood in terms of the $\mathrm{IP}_{3}$-mediated release mechanism. Finally, the finding that flash photolysis of caged $\mathrm{Ca}^{2+}$ does not initiate a wave, but the increase of $\mathrm{IP}_{3}$ in the cell is sufficient for wave generation (Leybaert et al., 1998) further supports the hypothesis for $\mathrm{IP}_{3^{-}}$ dependent ATP release.

We note that in all simulations presented so far we assumed that $\mathrm{IP}_{3}$ production is not significantly decreased by low intracellular $\mathrm{Ca}^{2+}$ concentrations (i.e. we set $\mathrm{a}_{5}=0$ ). If this is not the case, the strictly unidirectional coupling between the two subsystems is destroyed. However, we emphasize that the pattern of finite range of propagation remains intact. This is due to the fact that $\mathrm{Ca}^{2+}$ cannot invoke the production of $\mathrm{IP}_{3}$ by itself and lower cytosolic $\mathrm{Ca}^{2+}$ concentrations may hinder $\mathrm{IP}_{3}$ production, thus shrinking the oscillatory regime for the $\mathrm{Ca}^{2+}$ subsystem and diminishing excitability.

In two-dimensional astrocytic networks, circular waves with finite range of propagation can be observed. Such a wave is shown in Fig. 18. A focal ATP stimulus creates a wave of ATP and a wave of $\mathrm{Ca}^{2+}$, which both exhibit finite range. The radius of the stimulus is $7.5 \mu \mathrm{m}$ which means that one cell is stimulated, while the amplitude is $500 \mu \mathrm{M}$, which is less than the intracellular ATP content of one cell. The wave propagates for a radius of approximately $100 \mu \mathrm{m}$, (roughly 10 cell diameters), which compares well with experimental data. The maximum range is reached approximately 10 seconds after stimulation and then it starts to fade until the system returns to rest. 
Moreover, the speed of the ATP wave speed seems to be greater (around 40 - 50 $\mu \mathrm{m} / \mathrm{s}$ ) than the speed of the $\mathrm{Ca}^{2+}$ wave (around $15 \mu \mathrm{m} / \mathrm{s}$ ). It is interesting to observe that initially, the ATP wave spreads quickly and its attenuation is very large. On the contrary, the $\mathrm{Ca}^{2+}$ signal exhibits only modest attenuation, but it finally vanishes as shown in the last panel.

\section{DISCUSSION}

We developed a model that describes signal transduction mechanisms in a single astrocyte. The model describes ATP release, the production of $\mathrm{IP}_{3}$ from extracellular ATP stimuli and the subsequent secretion of $\mathrm{Ca}^{2+}$ to the cytosolic space due to the opening of $\mathrm{IP}_{3} \mathrm{R}$ channels. By incorporating the equations in a reaction-diffusion framework, wave generation, propagation and blocking were also studied. Two biological hypotheses about the mechanism of ATP release postulated in the literature were tested: $\mathrm{Ca}^{2+}$ - and $\mathrm{IP}_{3}$-dependent ATP release. Using this framework we studied the relationship between single-cell signal transduction mechanisms and wave propagation properties of coupled astrocytic networks.

The overall single-cell system consists of two subsystems, the ATP-IP ${ }_{3}$ nonexcitable driver system and the $\mathrm{Ca}^{2+}$ excitable subsystem. In the case of $\mathrm{Ca}^{2+}-$ dependent ATP release, the coupling between the two systems is bidirectional: the means of communication is the $\mathrm{IP}_{3}$ concentration (output of the driver, input to the driven) and the $\mathrm{Ca}^{2+}$ concentration (output of the driven, input to the driver). This is not the case however in the $\mathrm{IP}_{3}$-dependent ATP release, in which the coupling is unidirectional: the $\mathrm{IP}_{3}$ concentration is the only "signal" (output of the driver, input to the driven). The different coupling mechanisms were found to have a striking effect on the single cell and the coupled astrocytic network dynamics. 
$\mathrm{Ca}^{2+}$-dependent ATP release leads to excitable behavior and frequency-encoded oscillations in the entire system at the single-cell level, since these properties of the $\mathrm{Ca}^{2+}$ subsystem are inherited to the whole system. This behavior is observed only if $\mathrm{Ca}^{2+}$ has a sufficiently enhancing effect on ATP release, for when $\mathrm{Ca}^{2+}$ inhibits ATP release, the excitability of the system is destroyed. Infinite range of wave propagation is predicted in one-dimensional cell cultures when the $\mathrm{Ca}^{2+}$ positive feedback loop is sufficiently strong. The speed of the wave was found to scale with the square root of the diffusion coefficient of ATP, in agreement with the theory of reaction-diffusion equations. Significant $\mathrm{Ca}^{2+}$ feedback in $\mathrm{IP}_{3}$ production has a stabilizing effect, damping the oscillations due to the depression of $\mathrm{IP}_{3}$ production when $\mathrm{Ca}^{2+}$ exists in low concentrations.

In two dimensional homogeneous astrocytic cellular networks, ATP and $\mathrm{Ca}^{2+}$ spiral waves were generated from appropriate ATP stimulation patterns in the case where astrocytic coupling occurs predominantly through diffusion of extracellular ATP. These results suggest an alternative mechanism for the generation of the experimentally observed spiral waves than the one postulated by other authors, where spirals were a result of spatial inhomogeneities in conjunction with the $\mathrm{IP}_{3}$-mediated intercellular mode of communication.

On the other hand $\mathrm{IP}_{3}$-dependent ATP release leads to a unidirectional coupling of the non-excitable ATP-IP 3 master system, which drives the excitable $\mathrm{Ca}^{2+}$ subsystem. Thus, waves of finite range of propagation are generated in both the one and two dimensional homogeneous domains. The range of wave propagation depends on the amplitude of the ATP stimulus, but is always finite due to the single-cell level signal transduction mechanisms. For large enough ATP stimuli (higher than $1 \mathrm{mM}$ ), multiple $\mathrm{Ca}^{2+}$ waves with finite range of propagation can be generated, as a result of the 
prolonged high concentration of $\mathrm{IP}_{3}$ in the cytosol of the cells close to the point of stimulation.

In conclusion, the $\mathrm{IP}_{3}$-dependent $\mathrm{ATP}$ release hypothesis can help us understand finite range of propagation in terms of the single-cell mechanisms and the coupling between the $\mathrm{ATP}-\mathrm{IP}_{3}$ and the $\mathrm{Ca}^{2+}$ subsystems. We have not considered spatial inhomogeneities in our computational analysis. Thus, we show that finite range of propagation may not necessarily be a result of the geometry of the astrocytic network (namely the location of the ATP release sites and the dimensions of the reaction-free gap junctions through which $\mathrm{IP}_{3}$ can diffuse between astrocytes). The underlying mechanisms may inherently possess the ability to block waves from spreading throughout the whole cell culture. We have shown that in the case where a nonexcitable driver system $\left(\mathrm{ATP}-\mathrm{IP}_{3}\right)$ drives an excitable subsystem $\left(\mathrm{Ca}^{2+}\right.$ subsystem $)$ finite range of propagation can be predicted.

\section{ACKNOWLEDGEMENTS}

M.S. would like to thank Professors Andreas G. Boudouvis (National Technical University of Athens) and Kyriakos Zygourakis (Rice University) for many stimulating discussions regarding this work. N.V.M. would like to thank Professors Hans Othmer and Eric Newman (University of Minnesota) for introducing him to various aspects of astrocyte signaling. Both authors would like to thank the reviewers for their constructive criticism and their contribution to the improvement of this work. 


\section{APPENDIX}

We will show that, independently of the ATP release mechanism, the ATP-IP 3 subsystem cannot be excitable.

i) $\mathrm{Ca}^{2+}$-dependent ATP release:

From Eq. 10 and 11 the nullclines can be expressed in the H-I plane as follows:

$$
\begin{aligned}
& \mathrm{H}=\frac{\kappa_{8} \cdot \mathrm{c}_{7} \cdot \mathrm{v}_{7} \cdot \mathrm{F}(\mathrm{C})}{\mathrm{v}_{8}-\mathrm{c}_{7} \cdot \mathrm{v}_{7} \cdot \mathrm{F}(\mathrm{C})} \\
& \mathrm{H}=\frac{\mathrm{I} \cdot \mathrm{v}_{6} \cdot \kappa_{4}}{\mathrm{v}_{4} \cdot \frac{\mathrm{C}+\left(1-\alpha_{5}\right) \cdot \kappa_{5}}{\mathrm{C}+\kappa_{5}}-\mathrm{I} \cdot \mathrm{v}_{6}}
\end{aligned}
$$

The maximum degradation term is assumed to be greater than the maximum production term (to ensure that the concentration never blows up) thus the denominator in the expression $\mathrm{A} 1$ is always positive. For every $\mathrm{C}$ value, the nullcline $\mathrm{dH} / \mathrm{dt}=0$ is a horizontal line (independent of $\mathrm{IP}_{3}$ ) and the nullcline $\mathrm{dI} / \mathrm{dt}=0$ is a monotonically increasing function of I, with a singularity at:

$$
I=\frac{v_{4}}{v_{6}} \cdot \frac{C+\left(1-\alpha_{5}\right) \cdot \kappa_{5}}{C+\kappa_{5}}
$$

Thus, the ATP-IP 3 subsystem itself cannot have cubic shaped nullclines. Therefore, it cannot be excitable. Also it is interesting to note that it cannot even have multiple of solutions ( $\mathrm{C}$ always assumed to be constant).

ii) $\mathrm{IP}_{3}$-dependent $\mathrm{ATP}$ release

From Eq. 10 and the nullcline for $\mathrm{dH} / \mathrm{dt}=0$ can be expressed in the $\mathrm{H}-\mathrm{I}$ plane as follows:

$$
\mathrm{H}=\frac{\kappa_{8} \cdot \mathrm{c}_{7} \cdot \mathrm{v}_{7} \cdot \mathrm{G}(\mathrm{I})}{\mathrm{v}_{8}-\mathrm{c}_{7} \cdot \mathrm{v}_{7} \cdot \mathrm{G}(\mathrm{I})}
$$

The nullcline $\mathrm{dI} / \mathrm{dt}=0$ is the same as previously (Eq. A2). In this case the $\mathrm{dH} / \mathrm{dt}=0$ 
nullcline is a bell-shaped function of I since G(I) is bell-shaped too. Taking the derivative of the expression of Eq. A4, with respect to I we obtain:

$$
\frac{\mathrm{dH}}{\mathrm{dI}}=\frac{\mathrm{v}_{8} \cdot \kappa_{8} \cdot \mathrm{c}_{7} \cdot \mathrm{v}_{7} \cdot \mathrm{G}^{\prime}(\mathrm{I})}{\left(\mathrm{v}_{8}-\mathrm{c}_{7} \cdot \mathrm{v}_{7} \cdot \mathrm{G}(\mathrm{I})\right)^{2}}
$$

Since all parameters are positive and the denominator positive and finite, the roots of $\mathrm{dH} / \mathrm{dI}=0$ are exactly the same as the roots of $\mathrm{G}^{\prime}(\mathrm{I})$. Therefore, the $\mathrm{dH} / \mathrm{dt}=0$ nullcline has exactly as many extrema as $G(I)$. Thus, since $G^{\prime}(I)$ has one root the $\mathrm{dH} / \mathrm{dt}=0$ nullcline has only one extremum. Hence, this nullcline can never be cubic since a cubic nullcline has two extrema. The nullcline for $\mathrm{dI} / \mathrm{dt}=0$ is monotonic as explained before. Therefore, as in the case of $\mathrm{Ca}^{2+}$-dependent ATP release excitable behavior is not possible. Notice however that in this case we can have multiplicity of solutions for the ATP-IP ${ }_{3}$ system itself. 


\section{REFERENCES}

Allbritton, N. L., Meyer, T., Stryer, L., 1992. Range of messenger action of calcium ion and inositol 1,4,5-trisphosphate. Science. 258 (5089), 1812-1815.

Anderson, C. M., Swanson, R. A., 2000. Astrocyte glutamate transport: Review of properties, regulation, and physiological functions. Glia. 32 (1), 1-14. doi:10.1002/1098-1136(200010)32:1<1::AID-GLIA10>3.0.CO;2-W.

Atri, A., Amundson, J., Clapham, D., Sneyd, J., 1993. A single-pool model for intracellular calcium oscillations and waves in the Xenopus laevis oocyte. Biophys. J. 65 (4), 1727-1739.

Berridge, M. J., Bootman, M. D., Lipp, P., 1998. Calcium-a life and death signal. Nature. 395 (6703), 645-648. doi:10.1038/27094.

Bezprozvanny, I., Watras, J., Ehrlich, B. E., 1991. Bell-shaped calcium-response curves of $\operatorname{Ins}(1,4,5) \mathrm{P}_{3}$ and calcium-gated channels from endoplasmic reticulum of cerebellum. Nature (London). 351 (6329), 751-754. doi:10.1038/351751a0.

Bootman, M. D., Berridge, M. J., Taylor, C. W., 1992. All-or-nothing $\mathrm{Ca}^{2+}$ mobilization from the intracellular stores of single histamine-stimulated HeLa cells. J. Physiol. 450 (1), 163-178.

Brodal, P. 1998. The Central Nervous System: New York, Oxford University Press 1998. $675 \mathrm{p}$.

Carafoli, E., 2002. Calcium signaling: a tale for all seasons. Proc. Natl. Acad. Sci. USA. $99(3), 1115-1122$.

Charest, R., Prpic, V., Exton, J. H., Blackmore, P. F., 1985. Stimulation of inositol 
trisphosphate formation in hepatocytes by vasopressin, adrenaline and angiotensin II and its relationship to changes in cytosolic free $\mathrm{Ca}^{2+}$. Biochem. J. 227 (1), 79-90.

Charles, A., 1998. Intercellular calcium waves in glia. Glia. 24 (1), 39-49. doi:10.1002/(SICI)1098-1136(199809)24:1<39::AID-GLIA5>3.0.CO;2-W.

Charles, A. C., 1994. Glia-neuron intercellular calcium signaling. Dev. Neurosci. 16 (3/4), 196-206.

Charles, A. C., Dirksen, E. R., Merril, J. E., Sanderson, M. J., 1991. Intercellular signaling in glial cells: calcium waves and oscillations in response to mechanical stimulation and glutamate. Neuron. 6 (6), 983-992. doi:10.1016/0896-6273(91)90238-U.

Charles, A. C., Dirksen, E. R., Merrill, J. E., Sanderson, M. J., 1993. Mechanisms of intercellular calcium signaling in glial cells studied with dantrolene and thapsigargin. Glia. 7 (134-145).

Charles, A. C., Naus, C. C., Zhu, D., Kidder, G. M., Dirksen, E. R., Sanderson, M. J., 1992. Intercellular calcium signaling via gap junctions in glioma cells. J. Biol. Chem. 118 (1), 195-201.

Cornell-Bell, A. H., Finkbeiner, S. M., 1991. $\mathrm{Ca}^{2+}$ waves in astrocytes. Cell Calcium. $12(2 / 3)$, 185-204. doi:10.1016/0143-4160(91)90020-F.

Cornell-Bell, A. H., Finkbeiner, S. M., Cooper, M. S., Smith, S. J., 1990. Glutamate induces calcium waves in cultured astrocytes: long-range glial signaling. Science N. S. 247 (4941), 470-473.

Cotrina, M. L., Lin, J. H.-C., Alves-Rodrigues, A., Liu, S., Li, J., Azmi-Ghadimi, H., Kang, J., Naus, C. C. G., Nedergaard, M., 1998. Connexins regulate calcium 
signaling by controlling ATP release. Proc. Natl. Acad. Sci. USA. 95 (26), $15735-15740$.

Cunha, R. A., Sebastia, A. M., Ribeiro, J. A., 1998. Inhibition by ATP of hippocampal synaptic transmission requires localized extracellular catabolism by ecto-nucleotidases into adenosine and channeling to adenosine A1 receptors. J. Neurosci. 18 (6), 1987-1995.

D'Ambrosio, R., 2004. The role of glial membrane ion channels in seizures and epileptogenesis. Pharmacology \& Therapeutics. 103 (2), 95-108. doi:10.1016/j.pharmthera.2004.05.004.

Dani, J. W., Chernjavsky, A., Smith, S. J., 1992. Neuronal activity triggers calcium waves in hippocampal astrocyte networks. Neuron. 8, 429-440. doi:10.1016/0896-6273(92)90271-E.

De Young, G. W., Keizer, J., 1992. A single-pool inositol 1,4,5-triphosphate-receptorbased model for agonist-stimulates oscillations in $\mathrm{Ca}^{2+}$ concentration. PNAS. 89 (20), 9895-9899.

Dunwiddie, T. V., Diao, L., Proctor, W. R., 1997. Adenine nucleotides undergo rapid, quantitative conversion to adenosine in the extracellular space in rat hippocampus. J. Neurosci. 17 (20), 7673-7682.

Enkvist, M. O., McCarthy, K. D., 1992. Activation of protein kinase C blocks astroglial gap junction communication and inhibits the spread of calcium waves. J. Neurochem. 59, 519-526.

Ermentrout, B. 2002. Simulating, analyzing, and animating dynamical systems : a guide to XPPAUT for researchers and students: Philadelphia : Society for Industrial and Applied Mathematics, c2002. 
Fernandez-Garcia, G., Gomez-Gesteira, M., Muiiuzuri, A. P., Perez-Muiiuzuri, V., Perez-Villar, V., 1994. A method for spiral wave generation in the BelousovZhabotinsky reaction. Eur. J Phys. 15, 221-227.

Fife, P. C. 1979. Mathematical aspects of reacting and diffusing systems. New York: Springer-Verlag.

Finkbeiner, S., 1992. Calcium waves in astrocytes-filling in the gaps. Neuron. 8, 1101-1108. doi:10.1016/0896-6273(92)90131-V.

FitzHugh, R., 1960. Thresholds and plateus in the Hodgkin-Huxley nerve equations. J. Gen. Physiol. 43, 867-896.

Goldman, W. F., Yarowsky, P. J., Juhaszova, M., Krueger, B. K., Blaustein, M. P., 1994. Sodium/calcium exchange in rat cortical astrocytes. J. Neurosci. 14 (10), 5834-5843.

Graaf, R. A. d., Kranenburg, A. v., Nicolay, K., 2000. In vivo ${ }^{31}$ P-NMR diffusion spectroscopy of ATP and phosphocreatine in rat skeletal muscle. Biophys. J. $78,1657-1664$.

Guthrie, P. B., Knappenberger, J., Segal, M., Bennett, M. V. L., Charles, A. C., Kater, S. B., 1999. ATP released from astrocytes mediates glial calcium waves. J. Neurosci. 19 (2), 520-528.

Harris-White, M. E., Zanotti, S. A., Frautschy, S. A., Charles, A. C., 1998. Spiral intercellular calcium waves in hippocampal slice cultures. J. Neurophysiol. 79 (2), 1045-1052.

Hassinger, T. D., Atkinson, P. B., Strecker, G. J., Whalen, L. R., E., D. F., Koseel, A. H., Kater, S. B., 1995. Evidence for glutamate-mediated activation of hippocampal neurons by glial calcium waves. J. Neurobiol. 28, 159-170. 
Haydon, P. G., 2001. Glia: Listening and talking to the synapse. Nature Reviews, Neuroscience. 2 (3), 185-193. doi:10.1038/35058528.

Hofer, T., Politi, A., Heinrich, R., 2001. Intercellular Ca2+ Wave Propagation through Gap-Junctional Ca2+ Diffusion: A Theoretical Study. Biophys. J. 80 (1), 7587.

Hofer, T., Venance, L., Giaume, C., 2002. Control and Plasticity of Intercellular Calcium Waves in Astrocytes: A Modeling Approach. J. Neurosci. 22 (12), $4850-4859$

Holgado, A., Beauge, L., 1995. The $\mathrm{Na}^{+}-\mathrm{Ca}^{2+}$ exchange system in rat glial cells in culture - activation by external monovalent cations. Glia. 14 (2), 77-86. doi:10.1002/glia.440140202.

Innocenti, B., Parpura, V., Haydon, P. G., 2000. Imaging extracellular waves of glutamate during calcium signaling in cultured astrocytes. J. Neurosci. 20 (5), $1800-1808$

Jung, P., Cornell-Bell, A., Madden, K. S., Moss, F., 1998. Noise-induced spiral waves in astrocyte syncytia show evidence of self-organized criticality. J. Neurophysiol. 79 (2), 1098-1101.

Kang, J., Jiang, L., Goldman, S. A., Nedergaard, M., 1998. Astrocyte-mediated potentiation of inhibitory synaptic transmission. Nature Neuroscience. 1 (8), 683-692. doi:10.1038/3684

Kastritsis, C. H., Salm, A. K., McCarthy, K., 1992. Stimulation of the P2Y purinergic receptor on type 1 astroglia results in inositol phosphate formation and calcium mobilization. J. Neurochem. 58 (4), 1277-1284.

Kim, W. T., Rioult, M. G., Cornell-Bell, A. H., 1994. Glutamate-induced calcium 
signaling in astrocytes. Glia. 11 (2), 173-184. doi:10.1002/glia.440110211.

Lechleiter, J., Girard, S., Peralta, E., Clapham, D., 1991. Spiral calcium wave propagation and annihilation in Xenopus laevis oocytes. Science. 252 (5002), 123-126.

Lee, S. H., Magge, S., Spencer, D. D., Sontheimer, H., Cornell-Bell, A. H., 1995. Human epileptic astrocytes exhibit increased gap junction coupling. Glia. 15 (2), 195-202. doi:10.1002/glia.440150212.

Leibowitz, D. H., 1992. The glial spike theory. I. On an active role of neuroglia in spreading depression and migraine. Proceedings: Biological Sciences. 250 (1329), 287-295.

Leybaert, L., Paemeleire, K., Strahonja, A., Sanderson, M. J., 1998. Inositoltrisphosphate-dependent intercellular calcium signaling in and between astrocytes and endothelial cells. Glia. 24 (4), 398-407. doi:10.1002/(SICI)1098-1136(199812)24:4<398::AID-GLIA5>3.0.CO;2-R.

Li, Y.-X., Rinzel, J., 1994. Equations for $\mathrm{InsP}_{3}$ receptor-mediated $\left[\mathrm{Ca}^{2+}\right]_{\mathrm{i}}$ oscillations derived from a detailed kinetic model: a Hodgkin-Huxley like formalism. J. Theor. Biol. 166 (4), 461-473. doi:10.1006/jtbi.1994.1041.

Mishchenko, E. F., Rozov, N. K. 1980. Differential equations with small parameters and relaxation oscillations. Goodspeed FMC, translator: New York : Plenum Press, c1980.

Mouillac, B., Balestre, M.-N., Guillon, G., 1990. Positive feedback regulation of phospholiphase $\mathrm{C}$ by vasopressin-induced calcium mobilization in WRK1 cells. Cellular Signalling. 2 (5), 497-507. doi:10.1016/0898-6568(90)90046-D.

Murray, J. D. 1989. Mathematical Biology: Springer-Verlag. 
Nedergaard, M., 1994. Direct signaling from astrocytes to neurons in cultures of mammalian brain cells. Science. 263 (5154), 1768-1771.

Nedergaard, M., Ransom, B., Goldman, S. A., 2003. New roles for astrocytes: redefining the functional architecture of the brain. Trends Neurosci. 26 (10), 523-530. doi:10.1016/j.tins.2003.08.008.

Nett, W. J., Oloff, S. H., McCarthy, K. D., 2002. Hippocampal astrocytes in situ exhibit calcium oscillations that occur independent of neuronal activity. J. Neurophysiol. 87, 528-537.

Newman, E. A., 2001. Propagation of intercellular calcium waves in retinal astrocytes and Móller cells. J. Neurosci. 21 (7), 2215-2223.

Newman, E. A., Zahs, K. R., 1997. Calcium waves in retinal glial cells. Science. 275 (5301), 844-847. doi:10.1126/science.275.5301.844.

Neylon, C. B., Irvine, R. F., 1990. Synchronized repetitive spikes in cytoplasmic calcium in confluent monolayers of human umbilical vein endothelial cells. FEBS Lett. 275 (1/2), 173-176. doi:10.1016/0014-5793(90)81465-Z.

Orkand, R. K., Nicholls, J. G., Kuffler, S. W., 1966. Effect of nerve impulses on the membrane potential of glial cells in the central nervous system of amphibia. J. Neurophysiol. 29 (4), 788-806.

Parpura, V., Basarsky, T. A., Liu, F., Jeftinija, K., Jeftinija, S., Haydon, P. G., 1994. Glutamate-mediated astrocyte-neuron signalling. Nature. 369 (6483), 744747. doi:10.1038/369744a0.

Parri, H. R., Gould, T. M., Crunelli, V., 2001. Spontaneous astrocytic $\mathrm{Ca}^{2+}$ oscillations in situ drive NMDAR-mediated neuronal excitation. Nature Neuroscience. 4 (8), 803-812. doi:10.1038/90507. 
Putney, J. W. J., 1993. Excitement about calcium signaling in inexcitable cells. Science N. S. 262 (5134), 676-678.

Roderick, L., Berridge, M. J., Bootman, M. D., 2003. Calcium-induced calcium release. Curr. Biol. 13 (11), R425. doi:10.1016/S0960-9822(03)00358-0.

Salm, A. K., McCarthy, K. D., 1990. Norepinephrine-evoked calcium transients in cultured cerebral type 1 astroglia. Glia. 3 (6), 529-538. doi:10.1002/glia.440030612.

Sanderson, M. J., Charles, A. C., Boitano, S., Dirksen, E. R., 1994. Mechanisms and function of intercellular calcium signaling. Mol. Cell Endocrinol. 98 (2), 173187. doi:10.1016/0303-7207(94)90136-8.

Shao, Y., McCarthy, K. D., 1995. Receptor-mediated calcium signals in astroglia: multiple receptors, common stores and all-or-nothing responses. Cell Calcium. 17 (3), 187-196. doi:10.1016/0143-4160(95)90033-0.

Smrcka, A. V., Hepler, J. R., Brown, K. O., Sternweis, P. C., 1991. Regulation of polyphosphoinositide-specific phospholipase $\mathrm{C}$ activity by purified Gq. Science N. S. 251 (4995), 804-807.

Sneyd, J., Charles, A. C., Sanderson, M. J., 1994. A model for the propagation of intercellular calcium waves. Am. J. Physiol. Cell Physiol. 266 (1), C293C302.

Sneyd, J., Wetton, B. T., Charles, A. C., Sanderson, M. J., 1995. Intercellular calcium waves mediated by diffusion of inositol trisphosphate: a two-dimensional model. Am. J. Physiol. Cell Physiol. 268, C1537-C1545.

Starobin, J., Zilberter, Y. I., Starmer, C. F., 1994. Vunlerability in one-dimensional excitable media. Physica D. 70, 321-341. 
Takeda, M., Nelson, D. J., Soliven, B., 1995. Calcium signaling in cultured rat oligodendrocytes. Glia. 14 (3), 225-236. doi:10.1002/glia.440140308.

Tang, Y., Othmer, H., 1995. Frequency encoding in excitable systems with applications to calcium oscillations. PNAS. 92 (17), 7869-7873.

Venance, L., Stella, N., Glowinski, J., Giaume, C., 1997. Mechanism involved in initiation and propagation of receptor-induced intercellular calcium signaling in cultured rat astrocytes. J. Neurosci. 17 (6), 1981-1992.

Venance, V., Piomelli, D., Glowinski, J., Giaume, C., 1995. Inhibition by anandamide of gap junctions and intercellular calcium signalling in striatal astrocytes. Nature. 376, 590-594. doi:10.1038/376590a0.

Wang, S. S. H., Alousi, A. A., Thomson, S. H., 1995. The lifetime of inositol 1,4,5trisphosphate in single cells. J. Gen. Physiol. 105, 149-175.

Wang, Z., Haydon, P. G., Yeung, E. S., 2000. Direct observation of calciumindependent intercellular ATP signalling in astrocytes. Anal. Chem. 72 (9), 2001-2007. doi:10.1021/ac9912146.

Wiener, N., Rosenblueth, A., 1946. The mathematical formulation of the problem of conduction of impulses in a network of connected exitable elements specifically in cardiac muscle. Archivos Del Instituto De Cardiologia De Mexico. Ano 16; Tomo XVI (3,4), 205-265.

Wilkins, M., Sneyd, J., 1998. Intercellular Spiral Waves of Calcium. J. Theor. Biol. 191 (3), 299-308. doi:10.1006/jtbi.1997.0585.

Woods, N. M., Cuthbertson, K. S. R., Cobbold, P. H., 1987. Agonist-induced oscillations in cytoplasmic free calcium concentration in single rat hepatocytes. Cell Calcium. 8 (1), 79-100. doi:10.1016/0143-4160(87)90038-8. 
Yagodin, S. V., Holtzclaw, L., Russell, J. T., 1995. Subcellular calcium oscillators and calcium influx support agonist-induced calcium waves in cultured astrocytes. Mol. Cell Biochem. 149, 137-144.

Yagodin, S. V., Holtzclaw, L., Sheppard, C. A., Russell, J. T., 1994. Nonlinear propagation of agonist-induced cytoplasmic calcium waves in single astrocytes. J. Neurobiol. 25 (3), 265-280. doi:10.1002/neu.480250307. 


\section{FIGURE LEGENDS}

Figure 1. Signal transduction mechanisms. ATP binds to P2Y purinergic receptors in the membrane of the cell activating PLC which catalyzes $\mathrm{IP}_{3}$ production from $\mathrm{PIP}_{2}$. $\mathrm{IP}_{3}$ binds synergistically with $\mathrm{Ca}^{2+}$ in receptors on the surface of the endoplasmic reticulum (ER) and opens channels through which $\mathrm{Ca}^{2+}$ ions are secreted in the cytosol. $\mathrm{Ca}^{2+}$ has both promoting and inhibitory role on its own release. There is also a $\mathrm{Ca}^{2+}$ leak due to the large $\mathrm{Ca}^{2+}$ concentration gradient. $\mathrm{Ca}^{2+}$ is returned back to the ER by an ATP dependent pump. As a result of this cascade ATP is released in the extracellular space. There are two hypotheses about ATP release (shown in dashed lines): $\mathrm{Ca}^{2+}$-dependent or $\mathrm{IP}_{3}$-dependent ATP release.

Figure 2. Effect of the $\mathrm{Ca}^{2+}$ pump Michaelis Menten constant $\kappa_{3}$ on the excitability of the $\mathrm{Ca}^{2+}$ subsystem. The nullclines and vector field for the non-excitable case are shown in panel (a) $\left(\kappa_{3}=0.1 \mu \mathrm{M}\right)$. The cubic shaped nullcline corresponds to $\mathrm{dC} / \mathrm{dt}=$ 0 . The cubic shape is not sufficiently pronounced although there is a time scale separation. Thus, threshold phenomena are not observed as shown in the two transient simulations of panel (c), where the system starts from steady state conditions and a square pulse in time is imposed on $\left[\mathrm{Ca}^{2+}\right]$. The duration of the pulse is $5 \mathrm{~s}$ and the amplitude is $0.25 \mu \mathrm{M}$ for the lower curve and $0.75 \mu \mathrm{M}$ for the upper curve. Notice that the response in the two cases is proportional to the amplitude of stimuli. On the contrary, in the excitable case $\left(\kappa_{3}=0.05 \mu \mathrm{M}\right)$ the cubic shape is sufficiently pronounced (panel (b)) and sharp responses are observed for stimuli above a threshold as shown in panel (d), where again a square pulse of duration $5 \mathrm{~s}$ is imposed on the $\left[\mathrm{Ca}^{2+}\right]$ steady state concentration. The amplitude for the lower curve is $0.55 \mu \mathrm{M}$ and 
for the upper is $0.60 \mu \mathrm{M}$. Notice how large departure form the steady state is invoked by such a small difference between the two stimuli.

Figure 3. Effect of excitability in the bifurcation structure. Solid lines correspond to stable steady states, dashed, to unstable ones. Filled circles denote stable limit cycles, open circles unstable ones. In panels (a) and (c) all the attractors and the period of the limit cycles are shown for the non-excitable case $\left(\kappa_{3}=0.10 \mu \mathrm{M}\right.$, all other parameters as in table 1), with respect to $\mathrm{IP}_{3}$ concentration. In panels (b) and (d) the bifurcation diagram and the periods are shown for the excitable case $\left(\kappa_{3}=0.05 \mu \mathrm{M}\right.$, all other parameters as in table 1). In the excitable case frequency encoded oscillations are observed: the amplitude remains roughly constant but the period changes noticeably.

Figure 4. Interaction diagram for all the species in the $\mathrm{Ca}^{2+}$-dependent ATP release case. A feedback loop exists since $\mathrm{Ca}^{2+}$ can promote or inhibit ATP release.

Figure 5. Bifurcation diagrams for all four species in the $\mathrm{Ca}^{2+}$-dependent ATP release case. The bifurcation structure of the $\mathrm{Ca}^{2+}$ subsystem is inherited to ATP and $\mathrm{IP}_{3}$. Excitability and frequency encoding are observed for all species. All parameters as in tables $1-3$.

Figure 6. Effect of the extent of $\mathrm{Ca}^{2+}$ inhibition on ATP release as quantified by parameter $\mathrm{C}_{\max }$. For $\mathrm{C}_{\max }=0.5 \mu \mathrm{M}$ (panel a) the ATP steady state shifts to higher values, since the maximum positive feedback can be achieved, and starts decreasing. 
For $\mathrm{C}_{\max }=0.1 \mu \mathrm{M}$ (panel (b)) the inhibition is rather strong and an interesting folding appears.

Figure 7. Illustration of how the folding in Fig. 6 is created. The left column panels ((a), (c) and (e)) correspond to the hypothetical case where no $\mathrm{Ca}^{2+}$ inhibition on ATP release is present, while in the right column panels ((b), (d) and (f)) $\mathrm{Ca}^{2+}$ inhibition is included. Panels (e) and (f) present the $\mathrm{Ca}^{2+}$ bifurcation structure. We note the region of $\mathrm{Ca}^{2+}$ concentration where $\mathrm{Ca}^{2+}$ inhibits ATP release for the chosen value of $\mathrm{C}_{\max }$, as well as the region where $\mathrm{Ca}^{2+}$ enhances ATP release. Panel (a) shows the ATP bifurcation diagram, drawn as if no inhibition took place. Due to the inhibition at values higher than $\mathrm{C}_{\max }$ the branch above the dashed curve folds and creates the characteristic "lasso" shown in panel (b). The range of $\mathrm{IP}_{3}$ for which oscillations occur is the same as the range depicted in Fig. $4 \mathrm{~b}$.

Figure 8. Effect of the ATP Michaelis-Menten degradation rate threshold $\left(\kappa_{8}\right)$. A two parameter bifurcation diagram $\kappa_{8}-1 / \mathrm{v}_{6}$ is shown. Lines denote turning points and closed circles the Hopf point. Lower values of $\kappa_{8}$ shift the right turning point, where the oscillations cease, to lower $\mathrm{IP}_{6}$ degradation rates, thus shrinking the oscillatory regime. At roughly $\kappa_{8}=3.4 \mu \mathrm{M}$ the Hopf point is trapped inside the turning points and the oscillatory regime vanishes.

Figure 9. Effect of the increase of extracellular volume as quantified by parameter $\mathrm{c}_{7}$. In panel (a) $c_{7}=0.5$ and in (b) $c_{7}=0.25$ (larger extracellular volume for smaller values of $c_{7}$ ). The steady state of ATP shifts to lower values and the stable oscillatory 
regime vanishes. Thus, excitability is diminished by dilution because no significant amount of $\mathrm{IP}_{3}$ is produced in order to force the $\mathrm{Ca}^{2+}$ subsystem to the oscillatory regime.

Figure 10. Effect of $\mathrm{Ca}^{2+}$ feedback on $\mathrm{IP}_{3}$ production as quantified by parameter $\alpha_{5}$. In panel (a) the bifurcation structure for ATP is presented for $\alpha_{5}=1$, where the maximum possible feedback is obtained. In panel (b) a 2 parameter bifurcation diagram is shown. Solid lines show the turning points, while closed circles the Hopf point, from which the unstable periodic orbits of panel (a) arise. At around $\alpha_{5}=0.35$ the Hopf point is trapped inside the two turning points consequently the periodic orbits that arise are destroyed through homoclinic bifurcations before becoming stable.

Figure 11. Snapshots of traveling wave ATP and $\mathrm{Ca}^{2+}$ pulses of infinite range in onedimensional cell cultures for $\mathrm{Ca}^{2+}$-dependent ATP release and the extracellular mode of communication $\left(D_{\text {ATP }}=350 \mu \mathrm{m}^{2} / \mathrm{s} ; D_{\text {IP3 }}=0 \mu \mathrm{m}^{2} / \mathrm{s}\right)$. The corresponding time in seconds is given above each snapshot. Parameters as in tables $1,2,3$ except $\mathrm{v}_{6}=6.0$ $\mathrm{s}^{-1}$

Figure 12. Generation of a two armed spiral by applying point stimuli in ATP concentration. A circular wave is initiated at $\mathrm{t}=0 \mathrm{~s}$ (stimulus radius $7.5 \mu \mathrm{m}$, amplitude $100 \mu \mathrm{M})$. At $\mathrm{t}=73 \mathrm{~s}$ another stimulus generates a unidirectionally propagating front that evolves to a two armed ATP spiral. $\mathrm{D}_{\mathrm{ATP}}=350 \mu \mathrm{m}^{2} / \mathrm{s} ; \mathrm{D}_{\mathrm{IP} 3}=0$ $\mu \mathrm{m}^{2} / \mathrm{s} \mathrm{v}_{6}=6.0 \mathrm{~s}^{-1}$. Other parameters as in tables $1-3$. 
Figure 13. Interaction diagram in the $\mathrm{IP}_{3}$-dependent ATP release case. The system now consists a "master" non-excitable ATP-IP 3 subsystem, which drives the "slave" excitable $\mathrm{Ca}^{2+}$ subsystem.

Figure 14. Panels (a) and (b): bifurcation diagrams for ATP and $\mathrm{Ca}^{2+}$ in the $\mathrm{IP}_{3^{-}}$ dependent ATP release case parameters as in tables 1-3. A qualitatively different picture than in the $\mathrm{Ca}^{2+}$-dependent ATP release case is observed, since ATP exhibits a stable steady state; no frequency encoded oscillations or excitability is observed. The effect of strong $\mathrm{IP}_{3}$ inhibition is shown in panels (c) and $(\mathrm{d})\left(\mathrm{I}_{\max }=0.1 \mu \mathrm{M}\right)$ : the ATP steady state drops to lower values and the oscillatory regime is enlarged.

Figure 15. Snapshots of ATP and $\mathrm{Ca}^{2+}$ spatial profiles in a one-dimensional domain for the case of $\mathrm{IP}_{3}$-dependent $\mathrm{ATP}$ release generated by an ATP point stimulus (amplitude $500 \mu \mathrm{M}$, diameter $15 \mu \mathrm{m}$ which is slightly greater than a cell diameter) are shown. The corresponding time in seconds is given above each snapshot. $\mathrm{IP}_{3}$ degradation rate $\mathrm{v}_{6}=7.2 \mathrm{~s}^{-1}$, all other parameters as in tables 1-3.

Figure 16. Generation of a circular wave of finite range of propagation by applying a point ATP stimulus. At $\mathrm{t}=0 \mathrm{~s}$ a stimulus of radius $7.5 \mu \mathrm{m}$ (roughly 2 cells are stimulated) and amplitude $500 \mu \mathrm{M}$ on ATP is applied and creates an ATP and a $\mathrm{Ca}^{2+}$ wave that propagate for approximately $100 \mu \mathrm{m}$ (approximately 10 cell diameters). Notice the large decrease of the ATP signal in contrast to the modest attenuation of the $\mathrm{Ca}^{2+}$ signal, which is also experimentally observed. $\mathrm{IP}_{3}$ degradation rate: $\mathrm{v}_{6}=7.2$ 
$\mathrm{s}^{-1}$; all other parameters as in tables 1-3. 


\section{TABLES}

Table 1. Parameters for the $\mathrm{Ca}^{2+}$ subsystem of the $\mathrm{Li}$-Rinzel reduced model

\begin{tabular}{|c|c|c|c|}
\hline Symbol & Value & Units & Description \\
\hline $\mathrm{c}_{0}$ & 2.0 & $\mu \mathrm{M}$ & total $\mathrm{Ca}^{2+}$ concentration \\
\hline $\mathrm{c}_{1}$ & 0.185 & (dim/less) & ratio ER Volume / Cytoplasm Volume \\
\hline $\mathrm{v}_{1}$ & 6.0 & $\mathrm{~s}^{-1}$ & channel flux constant \\
\hline $\mathrm{v}_{2}$ & 0.11 & $\mathrm{~s}^{-1}$ & $\mathrm{Ca}^{2+}$ leak flux constant \\
\hline $\mathrm{v}_{3}$ & 0.9 & $\mu \mathrm{M} \cdot \mathrm{s}^{-1}$ & max pump flux \\
\hline$\kappa_{3}$ & 0.05 & $\mu \mathrm{M}$ & $\begin{array}{l}\text { constant in Hill function (this is the only } \\
\text { parameter different than Li-Rinzel where } \kappa_{3}=0.1 \text { ) }\end{array}$ \\
\hline$a_{2}$ & 0.2 & $(\mu \mathrm{M} \cdot \mathrm{s})^{-1}$ & $\begin{array}{l}\text { rate constant for } \mathrm{Ca}^{2+} \text { binding in inhibitory site } \\
\text { presence } \mathrm{IP}_{3}\end{array}$ \\
\hline $\mathrm{d}_{1}$ & 0.13 & $\mu \mathrm{M}$ & dissociation constant \\
\hline$d_{2}$ & 1.049 & $\mu \mathrm{M}$ & dissociation constant \\
\hline$d_{3}$ & 0.9434 & $\mu \mathrm{M}$ & dissociation constant \\
\hline $\mathrm{d}_{5}$ & 0.08234 & $\mu \mathrm{M}$ & dissociation constant \\
\hline
\end{tabular}


Table 2. Parameters for the $\mathrm{IP}_{3}$ balance

\begin{tabular}{llll}
\hline Symbol & Values & Units & Description \\
\hline $\mathrm{V}_{4}$ & 4.0 & $\mu \mathrm{M} \cdot \mathrm{s}^{-1}$ & max $\mathrm{IP}_{3}$ production \\
$\kappa_{4}$ & 0.3 & $\mu \mathrm{M}$ & constant in Michaelis-Menten func. for $\mathrm{H}^{*}$ \\
$\alpha_{5}$ & 0.0 & $($ dim/less $)$ & calcium feedback in $\mathrm{IP}_{3}$ production \\
$\kappa_{5}$ & 1.1 & $\mu \mathrm{M}$ & constant in Michaelis-Menten func. for $\mathrm{Ca}^{\ddagger}$ \\
$\mathrm{V}_{6}$ & 0.19 & $\mathrm{~s}^{-1}$ & $\mathrm{IP}_{3}$ degradation rate constant \\
\hline
\end{tabular}

from (Kastritsis et al., 1992) and (Charest et al., 1985) the Michaelis-Menten constant is about at $-\log ([\mathrm{ATP}])=5.5-6.5$ we use the values $10^{-6.5} \approx 0.3 \mu \mathrm{M}$.

$\$$ this is the same value as in (De Young and Keizer, 1992).

$\dagger$ range of values for this parameter can be found in (Wang et al., 1995). 
Table 3. Parameters for the ATP balance

\begin{tabular}{|c|c|c|c|}
\hline Symbol & Values & Units & Description \\
\hline $\mathrm{c}_{7}$ & 1.0 & (dim/less) & ratio Cytoplasm Vol./Extracellular Vol. \\
\hline $\mathrm{V}_{7}$ & 5.0 & $\mu \mathrm{M} \cdot \mathrm{s}^{-1}$ & max production rate \\
\hline $\mathrm{F}_{0}, \mathrm{G}_{0}$ & 0.05 & (dim/less) & constant (ATP feedback) \\
\hline $\mathrm{C}_{\max }, \mathrm{I}_{\max }$ & 1.5 & $\mu \mathrm{M}$ & constant (ATP feedback) $*$ \\
\hline $\mathrm{V}_{8}$ & 6.0 & $\mu \mathrm{M} \cdot \mathrm{s}^{-1}$ & max degradation rate \\
\hline$\kappa_{8}$ & 5.0 & $\mu \mathrm{M}$ & $\begin{array}{l}\text { constant in Michaelis-Menten function for ATP } \\
\text { degradation }\end{array}$ \\
\hline
\end{tabular}

* these values for $\mathrm{C}_{\max }, \mathrm{I}_{\max }$ are representative maximum values for intracellular $\mathrm{Ca}^{2+}$ and $\mathrm{IP}_{3}$ concentrations that have been experimentally observed. 


\section{FIGURES}

Extracellular Space

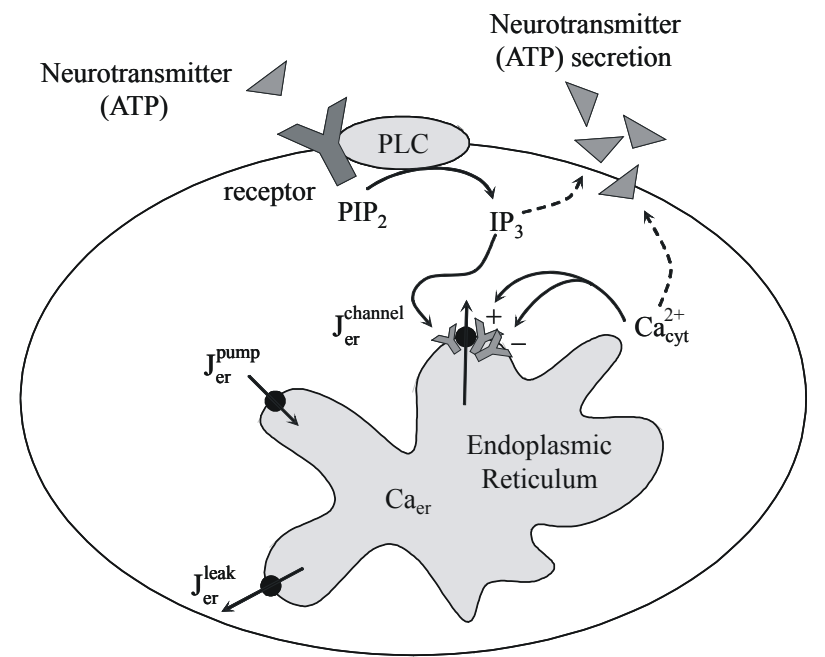

Figure 1 

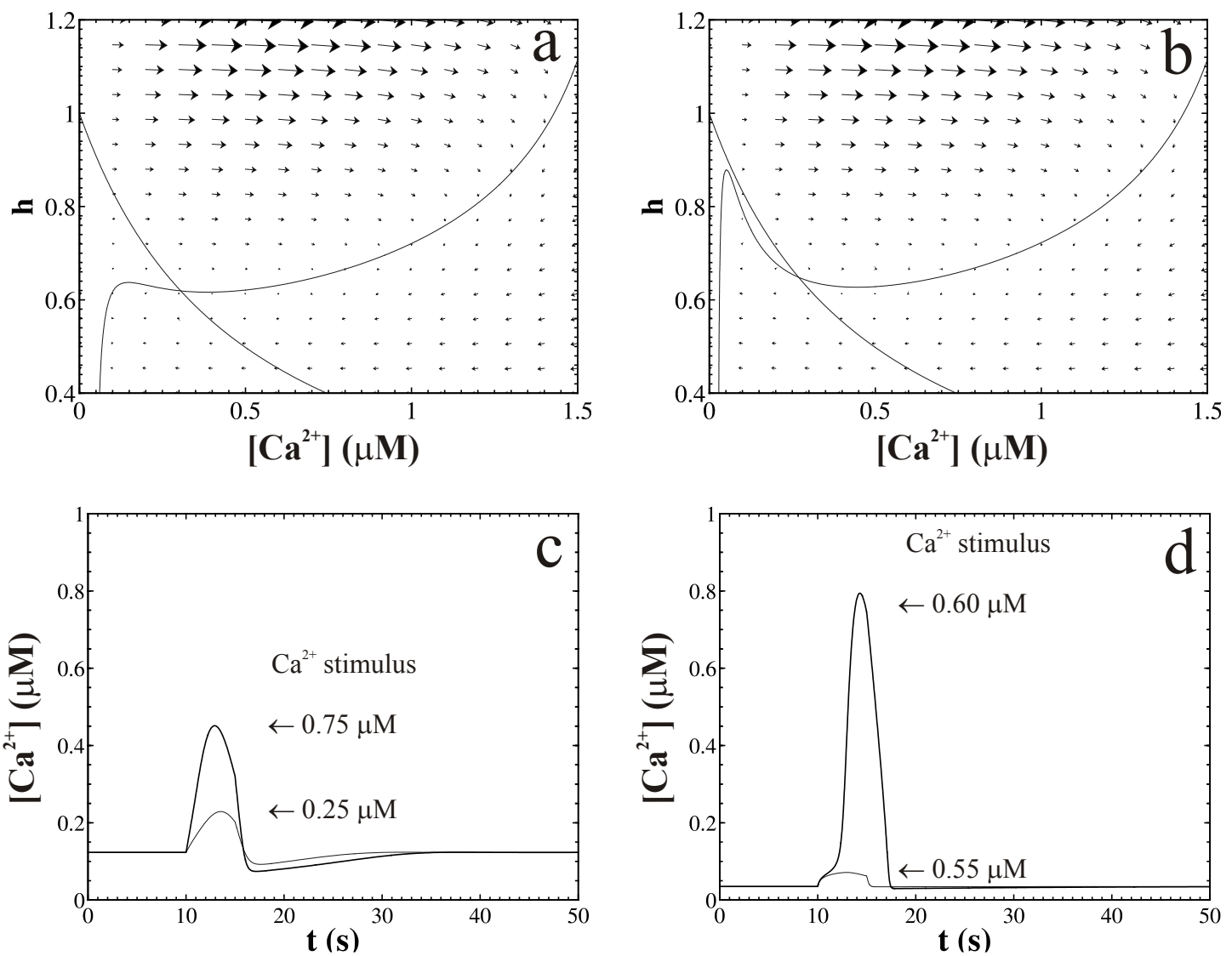

Figure 2 

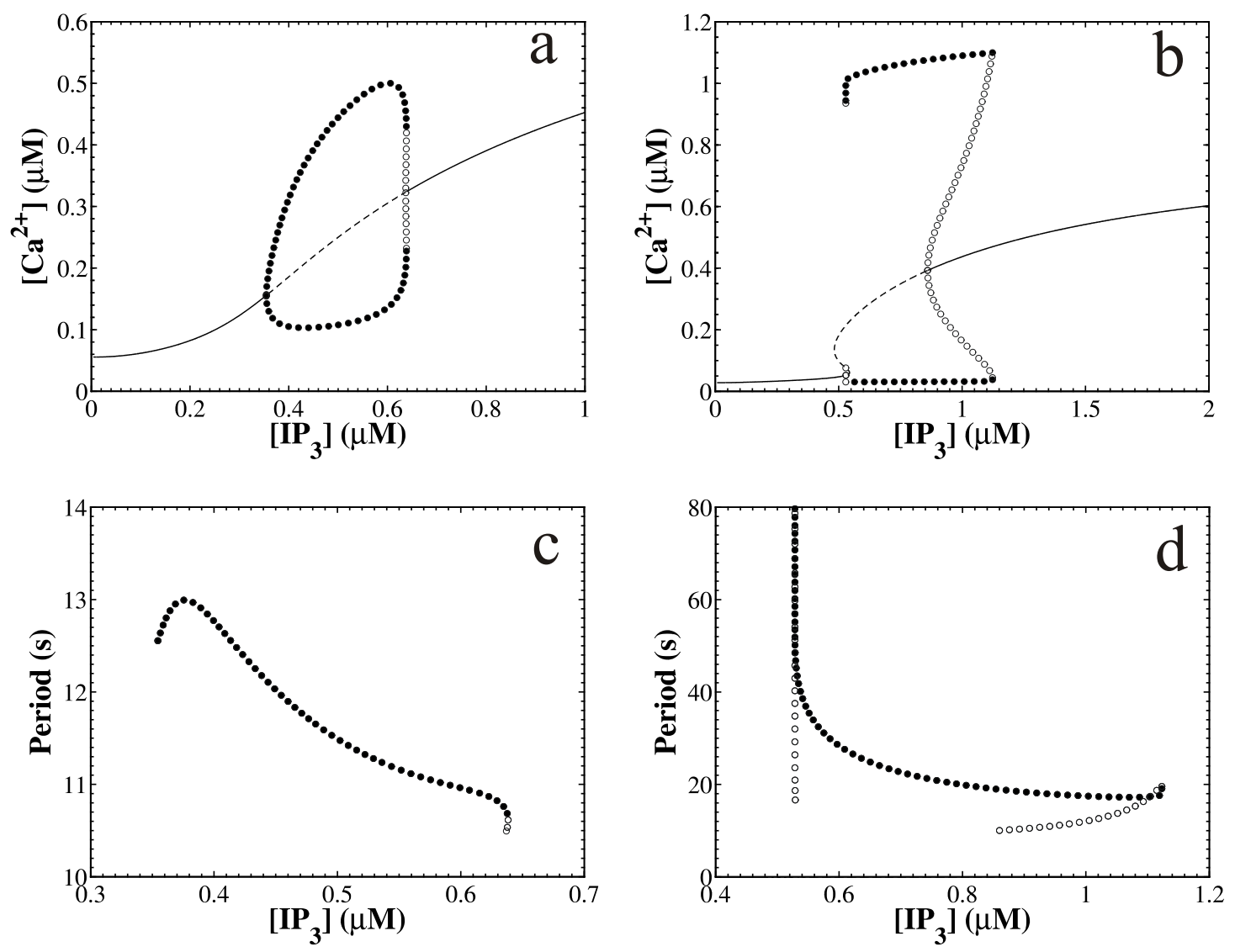

Figure 3 


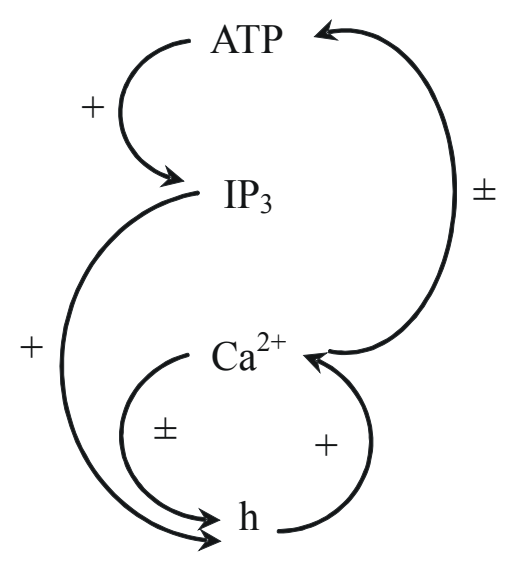

Figure 4 

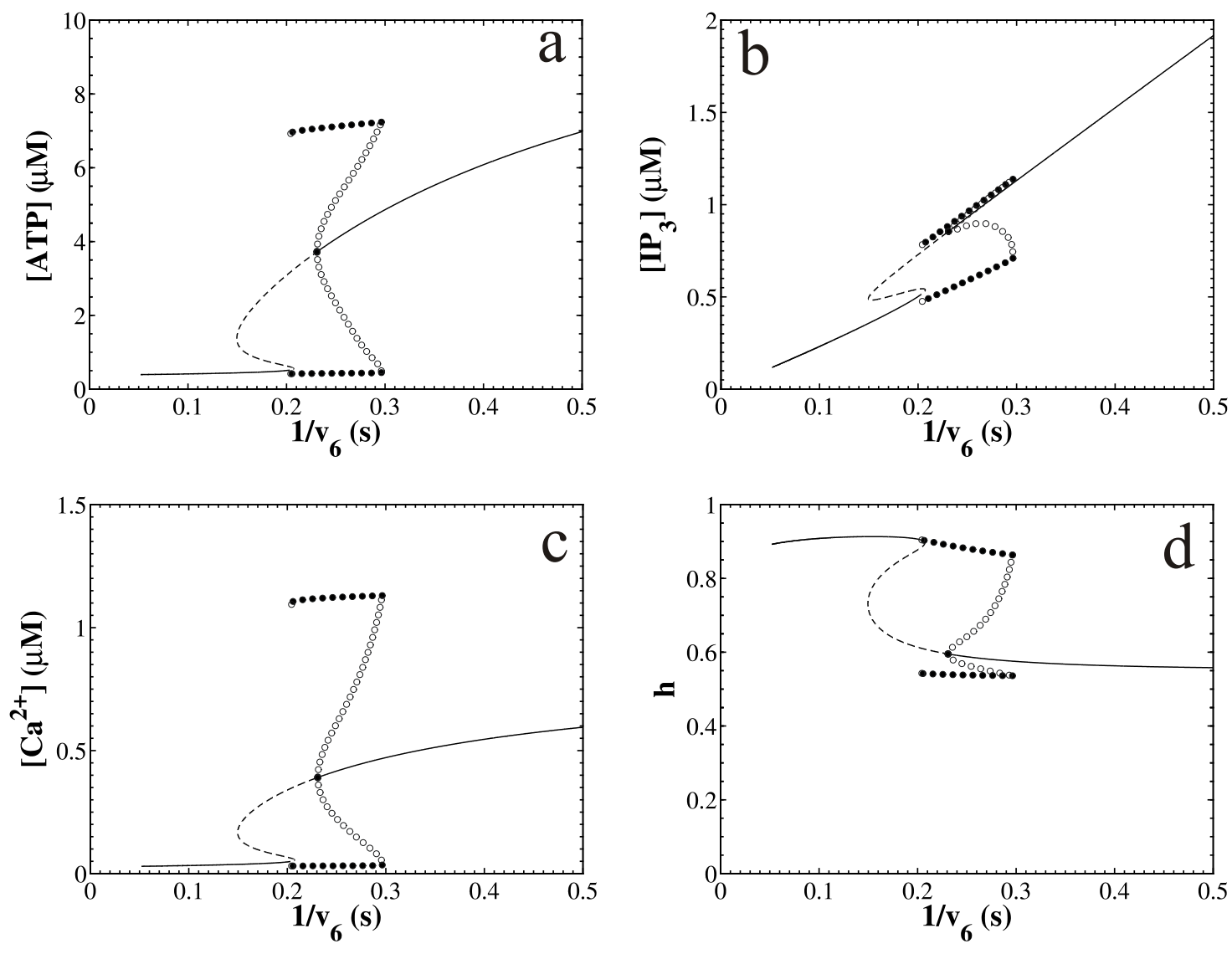

Figure 5 

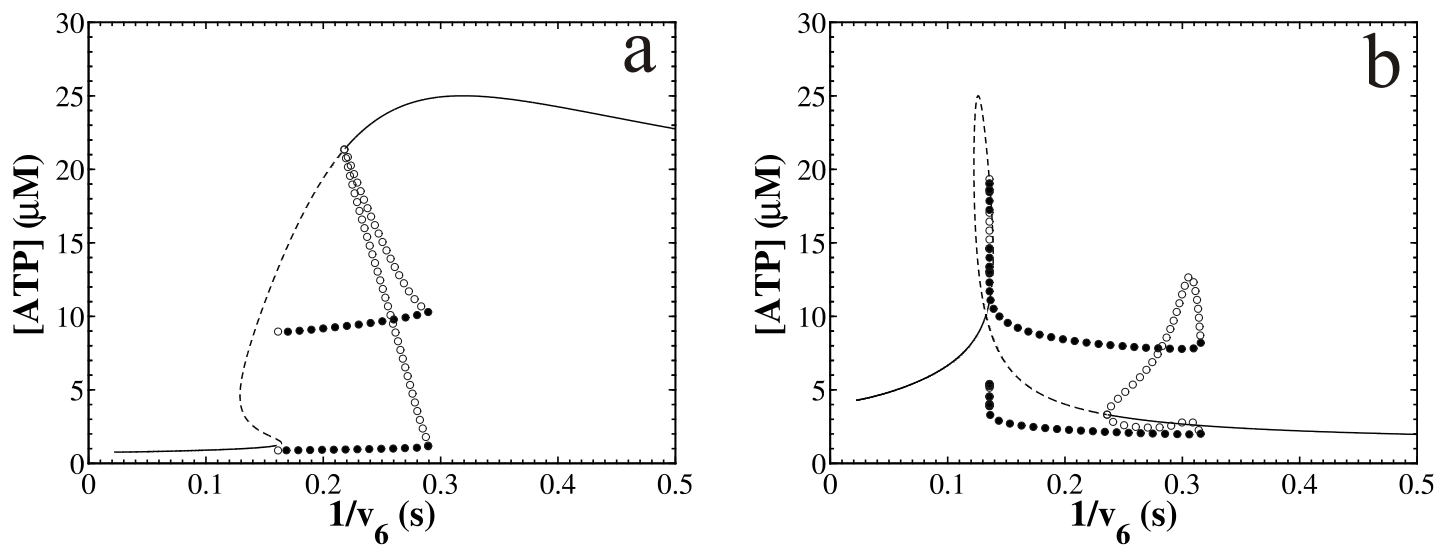

Figure 6 

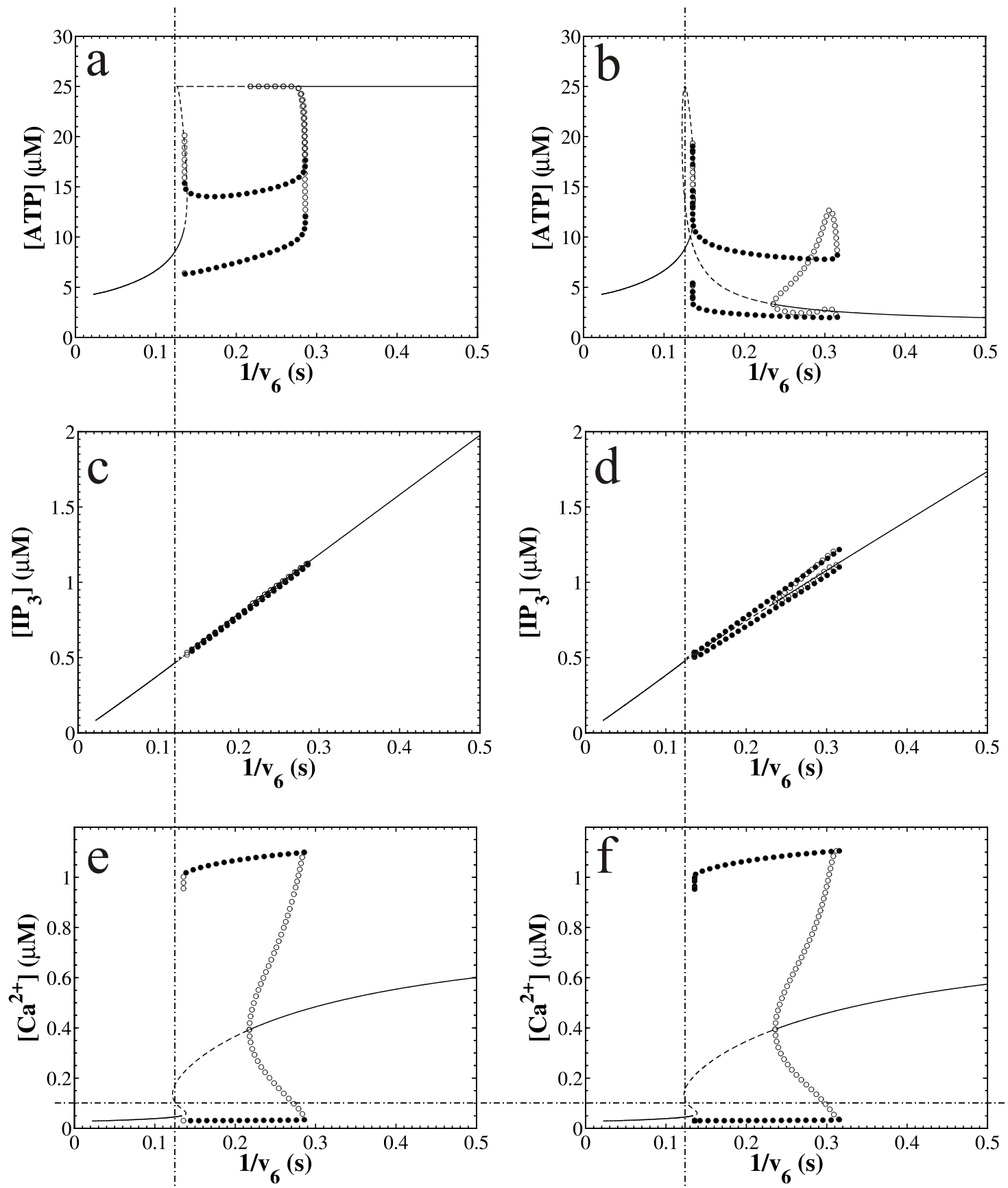

Figure 7 


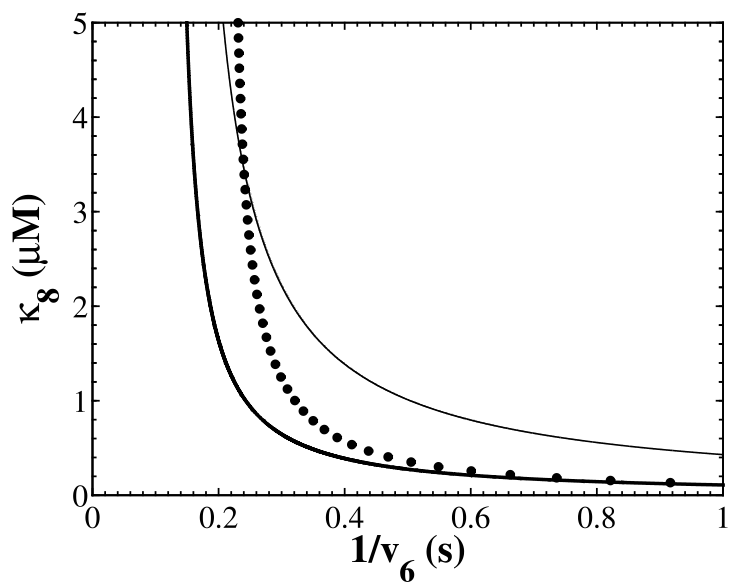

Figure 8 

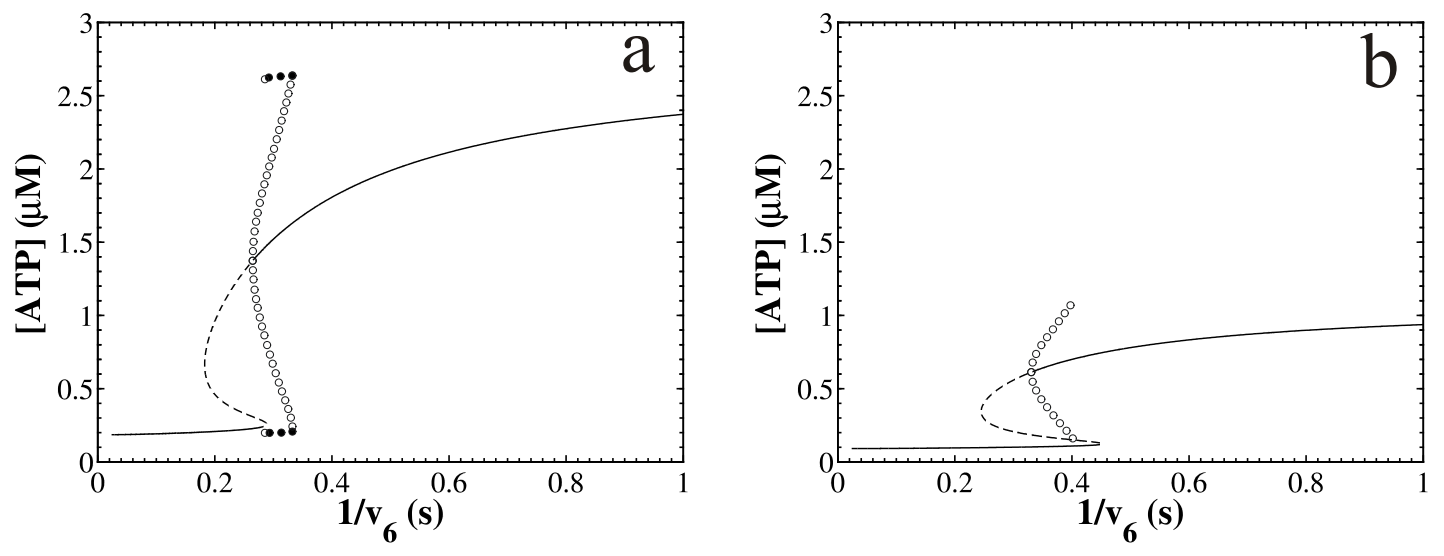

Figure 9 

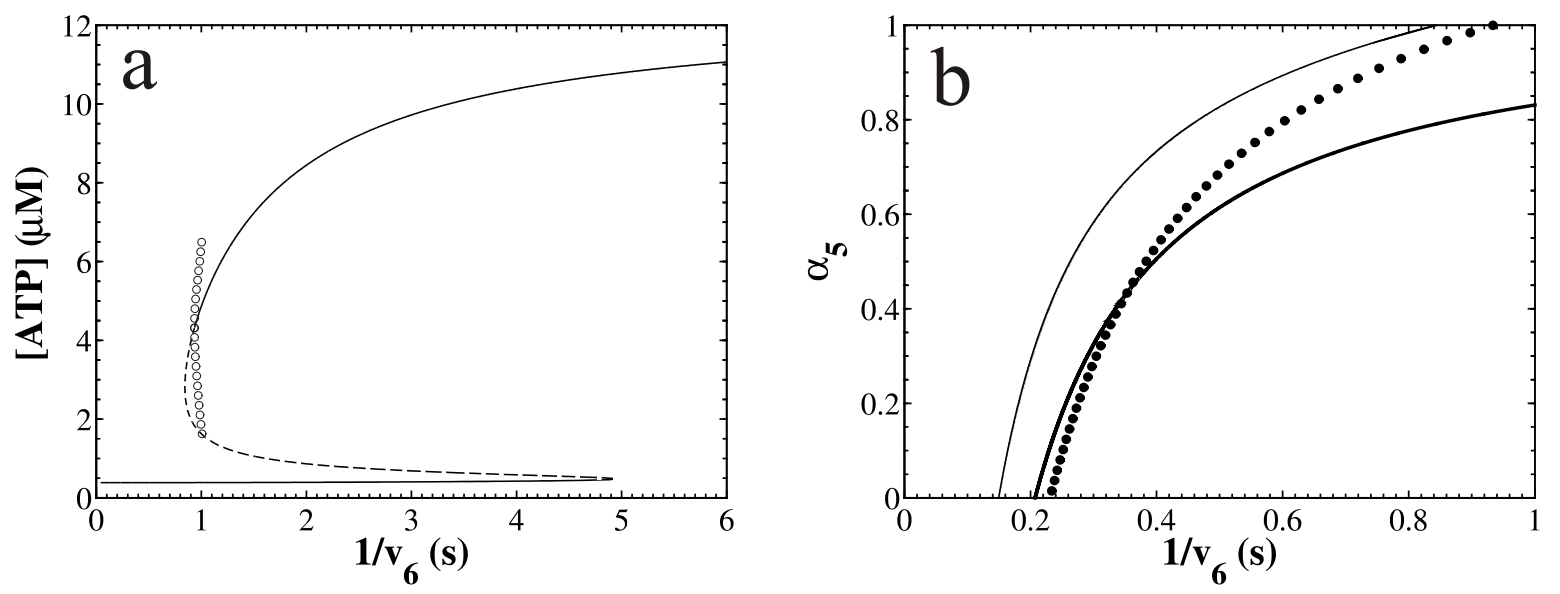

Figure 10 

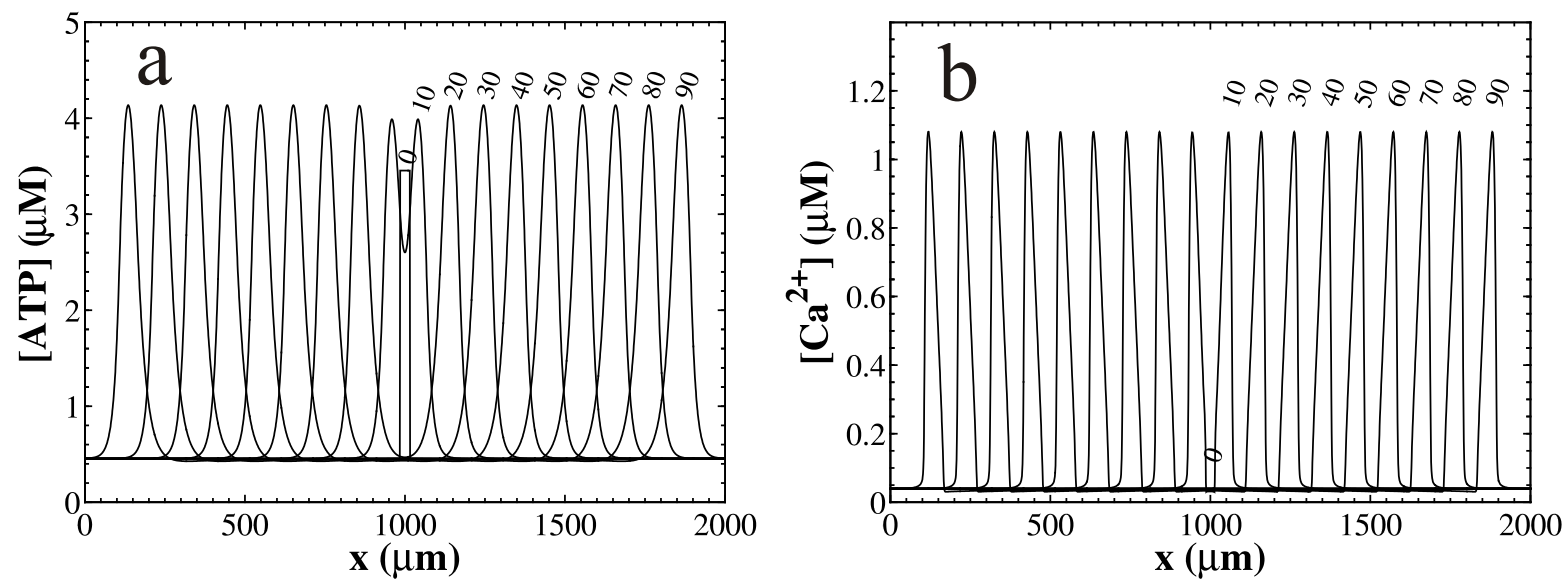

Figure 11 

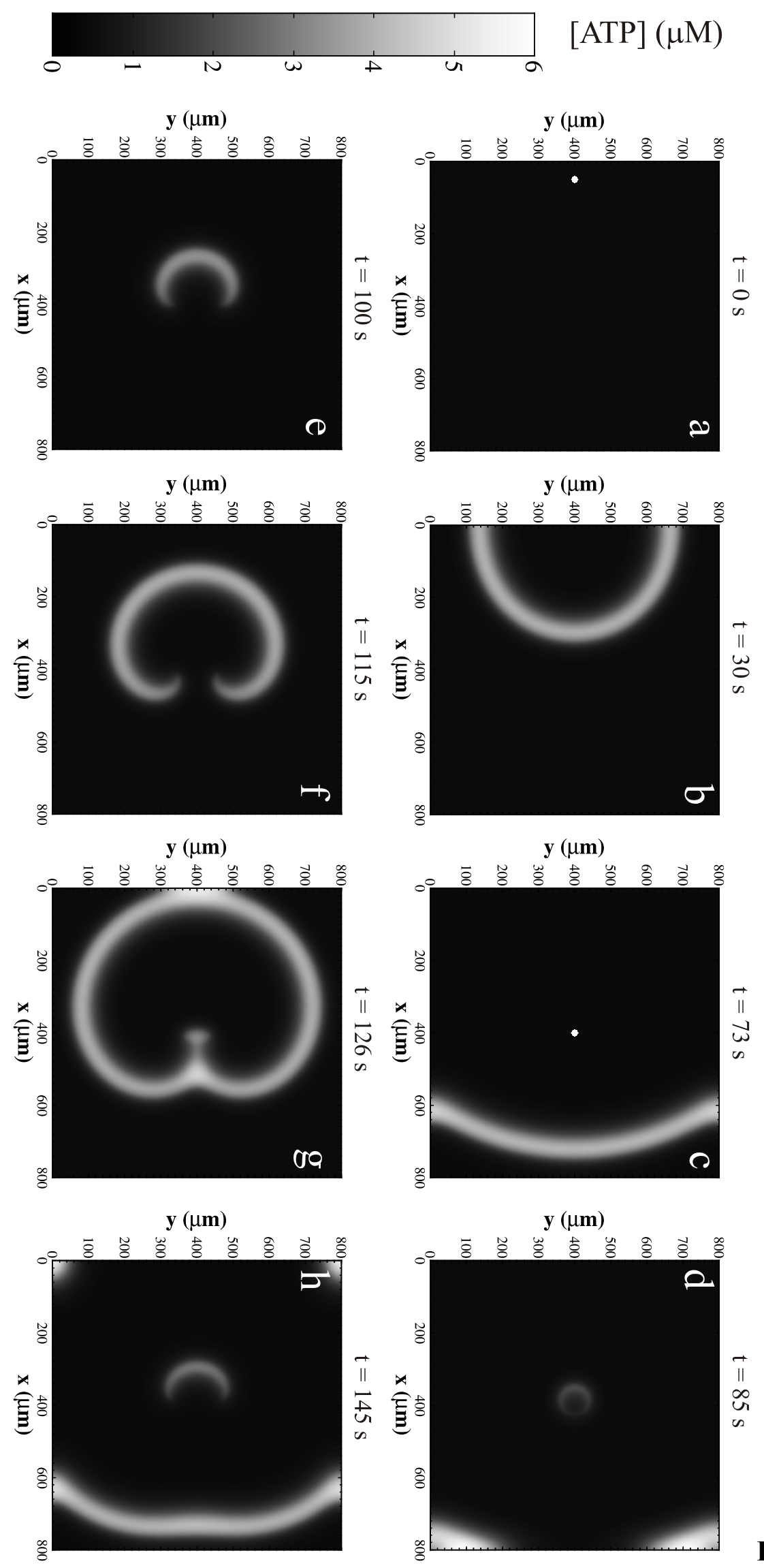

Figure 12 


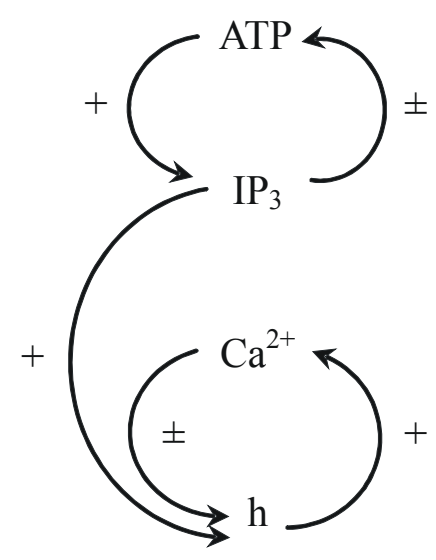

Figure 13 

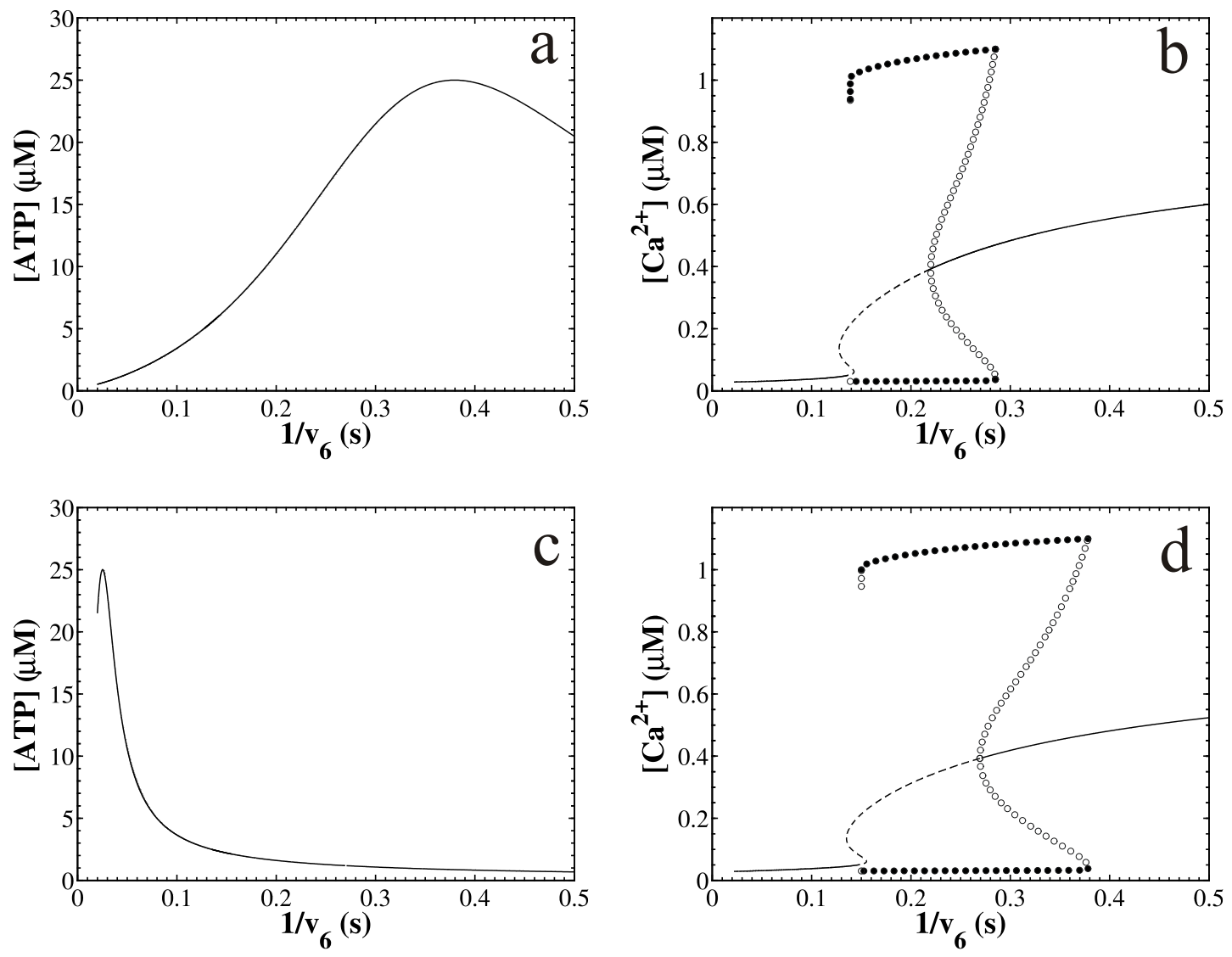

Figure 14 

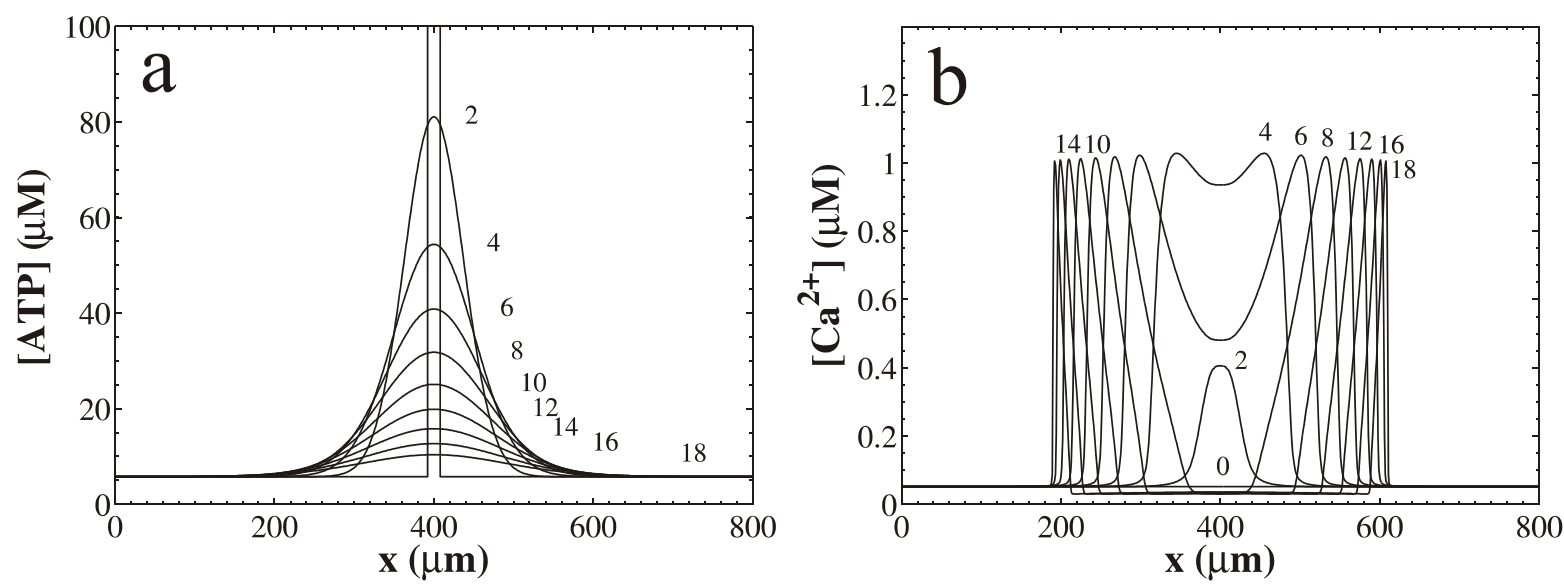

Figure 15 

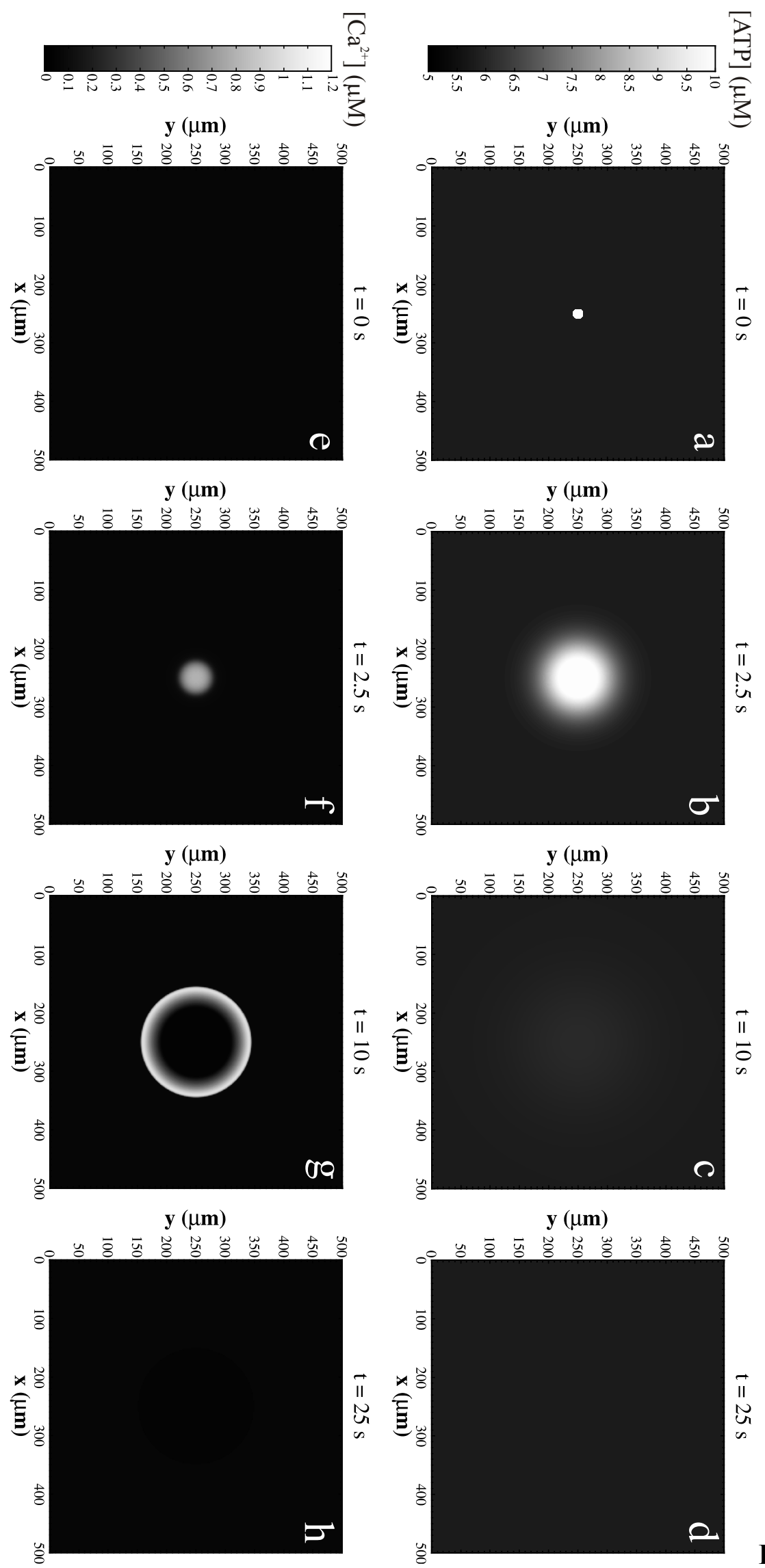

Figure 16 


\title{
Modeling of ATP-Mediated Signal Transduction and Wave Propagation in Astrocytic Cellular Networks
}

\author{
Michail Stamatakis, Nikos V. Mantzaris* \\ Department of Chemical and Biomolecular Engineering, Rice University, Houston, TX \\ 77005, USA \\ * Corresponding author. Department of Chemical and Biomolecular Engineering, Rice \\ University, Houston, TX 77005, USA. Phone: +1-713-348-2955; Fax: +1-713-348-5478; \\ e-mail: nman@rice.edu
}

\section{SUPPLEMENTAL MATERIAL}

\section{Distributed model: different parameter regimes}

The simulations presented here show that no wave generation and propagation occurs at the following parameter regimes: the oscillatory regime, the regime where stable oscillations coexist with an upper stable steady state and the regime where only an upper stable steady state exists. The parameter values used in simulations are as reported in tables 1, 2, 3 of the main paper, unless otherwise indicated.

\section{$\underline{\mathrm{Ca}^{2+} \text { dependent ATP case }}$}

In Fig. S1 $a, b$ results are shown for the oscillatory regime in the $\mathrm{Ca}^{2+}$-dependent ATP release case. The $\mathrm{IP}_{3}$ degradation rate is such that at the single cell level a stable limit cycle coexists with an unstable steady state (all parameters values as in tables 1, 2, 3 of main paper except $\mathrm{v}_{6}=4.7 \mathrm{~s}^{-1}$ ). The concentrations of ATP (panel (a)) and $\mathrm{Ca}^{2+}$ (panel (b)) are shown as contour plots with respect to space and time. Note that in this case all cells are oscillating indefinitely: if we observe the ATP concentration for a specific point in space, say $\mathrm{x}=10 \mu \mathrm{m}$, we see that through time the concentration raises (bright stripes) and falls (dark stripes) continuously (similar oscillations are observed for the other 
species). If and only if all cells were oscillating in phase, they would raise and lower their intracellular species' concentrations simultaneously, therefore, the contour plot would have the form of straight bright stripes parallel to the $\mathrm{x}$ axis, followed by dark stripes.

We now choose to impose a localized ATP stimulus at a point in time in which the minimum extracellular ATP concentration is observed, so that the response of the cells is more pronounced; if we stimulate when the cells "fire", no further "firing" is possible so the response would be minimal. The ATP stimulus incurs a shift in the phase of oscillations of nearby cells. This is shown in Fig. $1 a, b$ as a disturbance in the straight shape of the contours: we observe that in the point where the ATP stimulus was applied, the neighboring cells "fire" earlier, thus we get peak shaped contours, corresponding to the cells oscillating out of phase. However, for small times, the cells far away from the stimulus still oscillate in phase, that is why the contours of panels (a) and (b), for example, are straight parallel lines for $\mathrm{x}<300 \mu \mathrm{m}$. For large times (see the insets of panels (a) and (b)) this disturbance in the phase of oscillations is propagated to cells via diffusional coupling; this is not a chemical wave. Thus, the peaks of the contours are seem to smoothen out and the contours themselves tend to become straight lines. This means that the cells progressively return to the state observed before we stimulated them, namely the synchronized in phase oscillations. We see therefore that ATP diffusion and the bidirectional coupling of the $\mathrm{ATP}^{-\mathrm{IP}_{3}}$ driver system finally results in the resynchronization of the cells. Large ATP diffusion coefficients result in faster resynchronization.

Suppose now that we performed a similar simulation for a parameter set for which the stable limit cycle coexists with an upper stable steady state $\left(\mathrm{v}_{6}=4.0 \mathrm{~s}^{-1}\right)$. If we imposed 
the exact same stimulus on an oscillating astrocytic network at the time in which the minimum ATP concentration was observed we would observe a response similar to that described previously (shown in Fig. S1 $a$ and $b$ ). No cells can equilibrate to the upper steady state, because the $\mathrm{IP}_{3}$ concentration rapidly increases as a result of ATP stimulation, thus it does not stay for sufficient time in levels appropriate for the cells to rest to the steady state.

On the other hand, a different behavior is observed if a focal ATP stimulus is imposed on the upper steady state, which coexists with a stable limit cycle. As shown in Fig. S1 $b$, $c\left(\mathrm{v}_{6}=4.0 \mathrm{~s}^{-1}\right)$, here the astrocytic network initially rests at the upper steady state, no cells oscillate. In this case, the ATP stimulus merely produces a response, it fades out and no wave is generated. As the ATP stimulus vanishes, the $\mathrm{IP}_{3}$ concentration does not remain for sufficient time within levels able to initiate oscillations of the $\mathrm{Ca}^{2+}$ subsystem, thus no cells can enter the oscillatory regime. This behavior is also seen in the case in which the upper stable steady state is the only attractor (for large $1 / \mathrm{v}_{6}$ values). So, if we simulate an astrocytic network resting at the only stable upper steady state, the responses produced will be qualitatively identical to that shown in Fig. S1 $c, d$. This is also intuitively expected, since the $\mathrm{Ca}^{2+}$ subsystem is already excited and it cannot be further triggered to release more $\mathrm{Ca}^{2+}$, thus producing a response.

\section{$\underline{\mathrm{IP}_{3}} \underline{\underline{\text { dependent ATP }} \text { case }}$}

Similar simulations were performed for the $\mathrm{IP}_{3}$-dependent ATP release case. In Fig. $\mathrm{S} 2 a, b$, an astrocytic network is perturbed from a limit cycle, which is the only stable attractor for this parameter regime (all parameters values as in tables 1,2,3 of main paper except $\mathrm{v}_{6}=7.19 \mathrm{~s}^{-1}$ ). Note that in this case, the ATP $-\mathrm{IP}_{3}$ master system equilibrates and 
only the $\mathrm{Ca}^{2+}$ subsystem (species $\left.\mathrm{Ca}^{2+}, \mathrm{h}\right)$ oscillates, in contrast to the precious case $\left(\mathrm{Ca}^{2+}\right.$ dependent ATP release), in which all species oscillated. We now impose a localized ATP stimulus at a point in time when the intracellular $\mathrm{Ca}^{2+}$ concentration is at the minimum value (this is done in order to produce the most pronounced response as argued previously). In contrast to the $\mathrm{Ca}^{2+}$-dependent ATP release case, here the ATP concentration returns to the equilibrium value after the stimulus is imposed. However, the $\mathrm{IP}_{3}$ that is produced, triggers the $\mathrm{Ca}^{2+}$ subsystem, resulting in a shift of the phase of $\mathrm{Ca}^{2+}$ oscillations of nearby cells. After a while, the $\mathrm{IP}_{3}$ concentration returns to the steady state as the ATP concentration did, yet, the shift in the phase of $\mathrm{Ca}^{2+}$ oscillations remains: notice that, for large times (inset of Fig. S2 b), the contours of $\mathrm{Ca}^{2+}$ concentration do not tend to smoothen out to straight lines. This means that the cells continue oscillating asynchronously, even after a long time has passed from the moment they were stimulated. This is another qualitative difference from the previous case $\left(\mathrm{Ca}^{2+}\right.$-dependent ATP release) and can be attributed to two factors: 1) the unidirectional coupling between the ATP-IP ${ }_{3}$ driver system and the $\mathrm{Ca}^{2+}$ subsystem and 2) the fact that within the $\mathrm{Ca}^{2+}$ subsystem no coupling exists $\left(\mathrm{Ca}^{2+}\right.$ does not diffuse).

Moreover, suppose that the astrocytic network now exists in the parameter regime where the oscillations coexist with the stable upper steady state. Provided that the network is perturbed from the limit cycle, as before, the same qualitative behavior will be observed.

On the contrary, if the astrocytic network initially rests in the upper steady state and a localized ATP stimulus is imposed, no response is essentially triggered, the ATP stimulus fades and apparently no wave generation occurs as shown in Fig. S2, $c, d\left(\mathrm{v}_{6}=4.17 \mathrm{~s}^{-1}\right)$. 
In this simulation, the upper steady state coexists with the limit cycle, but no cells enter the limit cycle, since the $\mathrm{IP}_{3}$ does not remain in high enough levels for sufficient time, so as to initiate oscillating responses from the $\mathrm{Ca}^{2+}$ subsystem.

If the same simulation was performed in the parameter regime in which the only stable attractor is an upper stable steady state (large $1 / \mathrm{v}_{6}$ values), then similarly to the case just described, no response would be triggered. This can be attributed to the fact that the $\mathrm{Ca}^{2+}$ subsystem is already excited, it cannot be further excited, thus no response is initiated and no wave generation is observed. Thus, the behavior of the network would be qualitatively the same to that displayed in Fig. S2 $c, d$.

\section{Distributed model: modes of intercellular communication}

\section{$\underline{\mathrm{Ca}^{2+} \text { dependent ATP case }}$}

The effect of diffusion of both messenger species $\left(\mathrm{ATP}\right.$ and $\left.\mathrm{IP}_{3}\right)$ was investigated and was found to be negligible for the experimentally reported values of $\mathrm{IP}_{3}$ diffusion coefficient $\left(D_{\mathrm{IP} 3}=280 \mu \mathrm{m}^{2} / \mathrm{s}\right)$. This is shown in Fig. S3, in which panel (a) presents the case where only ATP diffuses and panel (b) the case where both ATP and $\mathrm{IP}_{3}$ diffuse. Contour plots of the $\mathrm{Ca}^{2+}$ concentrations with respect to space and time are shown and are found to be nearly indistinct. This happens because the dynamics of $\mathrm{IP}_{3}$ production are strictly dependent on ATP, which receives feedback from $\mathrm{Ca}^{2+}$. Thus the two species that dominate in shaping the response of the system are $\mathrm{Ca}^{2+}$ and $\mathrm{ATP}$; $\mathrm{IP}_{3}$ is just used as an intermediate messenger. To support this hypothesis, we performed simulations in which we used values for $\mathrm{IP}_{3}$ diffusion several times larger than the experimentally measure value (for example 10-fold). If the above reasoning was correct, then an increase 
of the $\mathrm{IP}_{3}$ diffusion coefficient would not be able to change wave propagation characteristics, namely the speed of the wave. This is indeed what was observed: for $\mathrm{D}_{\mathrm{IP} 3}$ $=0,280,2800 \mu \mathrm{m}^{2} / \mathrm{s}$ the speed of the wave remains constant at approximately $10 \mu \mathrm{m} / \mathrm{s}$. However we have to note that very large values of the $\mathrm{IP}_{3}$ diffusion coefficient, result in the transient flattening of the $\mathrm{IP}_{3}$ concentration profile. This means that the $\mathrm{IP}_{3}$ produced by the ATP stimulus rapidly diffuses away, consequently no wave generation is observed as the $\mathrm{Ca}^{2+}$ subsystem cannot be excited.

\section{$\underline{\mathrm{IP}_{3}} \underline{\text { dependent ATP case }}$}

For the $\mathrm{IP}_{3}$ dependent ATP release hypothesis, the effect of $\mathrm{IP}_{3}$ diffusion was also found to be negligible for the experimentally reported values of $D_{I P 3}$. In Fig. S4, a wave is generated and is found to have nearly the same range of propagation in the cases where only ATP diffuses (panel (a)), or both ATP and $\mathrm{IP}_{3}$ diffuse (panel (b)). In this case, the approximately same range of propagation can be attributed to the fact that ATP diffusion is the predominant mode of intercellular communication. Indeed, for significantly larger values of the $\mathrm{IP}_{3}$ diffusion coefficient, larger ranges of propagation are observed, provided that the ATP stimulus is sufficiently high. The large magnitude of ATP stimulus is required, because larger values for $\mathrm{D}_{\mathrm{IP} 3}$ tend to flatten the $\mathrm{IP}_{3}$ concentration profile quickly. Therefore, if large quantities of $\mathrm{IP}_{3}$ are produced (from a large ATP stimulus), $\mathrm{IP}_{3}$ will diffuse rapidly, resulting in a larger range of propagation. On the other hand an insufficient ATP stimulus will produce a small amount of $\mathrm{IP}_{3}$ which will be quickly flattened through diffusion, resulting in a smaller range of propagation. 


\section{FIGURES}
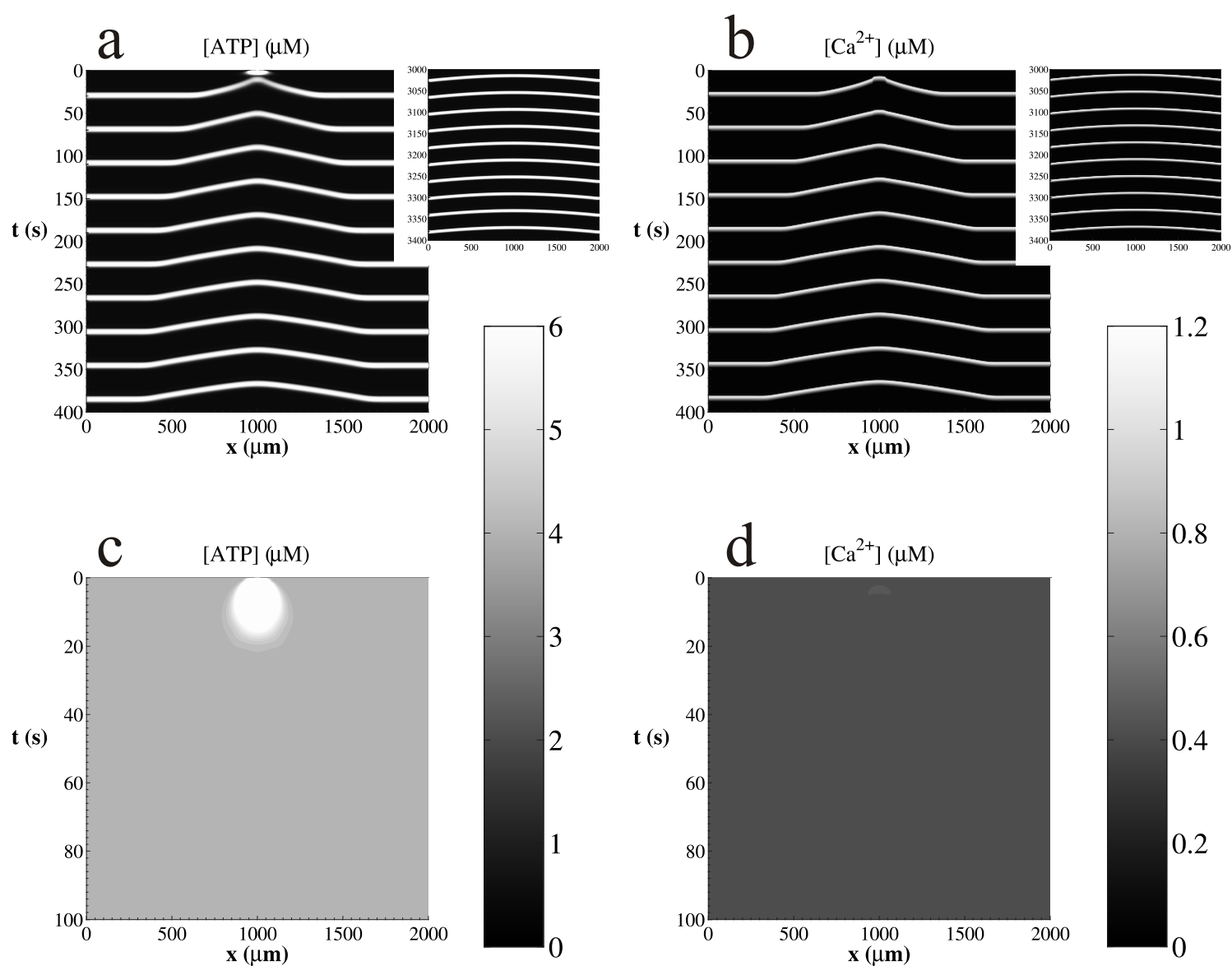

Figure S1: $\mathrm{Ca}^{2+}$ dependent ATP release case: panels (a), (b): imposing a localized ATP stimulus on the oscillating astrocytic network causes a shift in the phase of nearby cells $\left(\mathrm{v}_{6}=4.7 \mathrm{~s}^{-1}\right)$. The disturbance is propagated to cells in a diffusive manner; this is not a chemical wave. Simulations indicate that diffusional coupling tends to smoothen out the phase gradient (the insets show the ATP and $\mathrm{Ca}^{2+}$ concentrations for large times). Panels (b), (c): imposing a localized ATP stimulus on the upper steady state merely produces a response. In this case $\mathrm{v}_{6}=4.0 \mathrm{~s}^{-1}$, thus the steady state coexists with a stable limit cycle. Parameters values (except $\mathrm{v}_{6}$ ) as in tables $1,2,3$. Only ATP diffuses $\mathrm{D}_{\text {ATP }}=350 \mu \mathrm{m}^{2} / \mathrm{s}$. The stimulus is a square pulse in space of width $15 \mu \mathrm{m}$ for $a$ and $b(30 \mu \mathrm{m}$ for $c$ and $d)$ and amplitude $200 \mu \mathrm{m}$ imposed at $\mathrm{x}=1000 \mu \mathrm{m}$ at $\mathrm{t}=0 \mathrm{~s}$. 

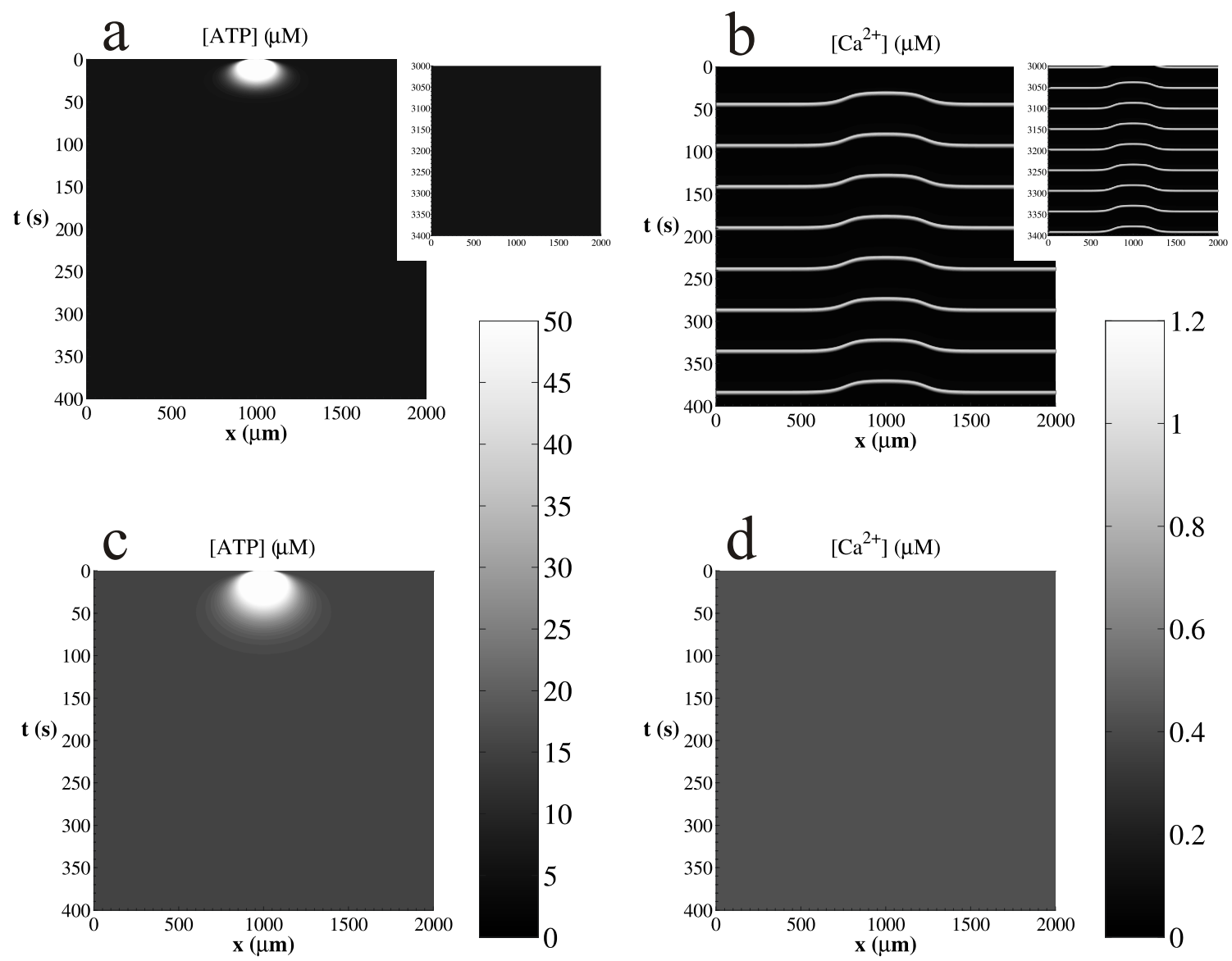

Figure S2: $\mathrm{IP}_{3}$ dependent ATP release case: panels (a), (b): imposing a localized ATP stimulus on the oscillating astrocytic network causes a shift in the phase of nearby cells $\left(\mathrm{v}_{6}=7.19 \mathrm{~s}^{-1}\right)$. Note that in this case only the $\mathrm{Ca}^{2+}$ subsystem oscillates. In contrast with the previous case $\left(\mathrm{Ca}^{2+}\right.$ dependent ATP release) the ATP stimulus fades quickly and the phase gradient is not smoothed out since no diffusional coupling exists in the $\mathrm{Ca}^{2+}$ subsystem. Panels (c), (d): imposing a localized ATP stimulus on the upper steady state which coexists with a limit cycle, merely produces a response $\left(\mathrm{v}_{6}=4.17 \mathrm{~s}^{-1}\right)$. Parameters values (except $\left.\mathrm{v}_{6}\right)$ as in tables 1,2 , 3. Only ATP diffuses $D_{\text {ATP }}=350 \mu \mathrm{m}^{2} / \mathrm{s}$. The stimulus is a square pulse in space of width $30 \mu \mathrm{m}$ and amplitude $1000 \mu \mathrm{m}$ imposed at $\mathrm{x}=1000 \mu \mathrm{m}$ at $\mathrm{t}=0 \mathrm{~s}$. 

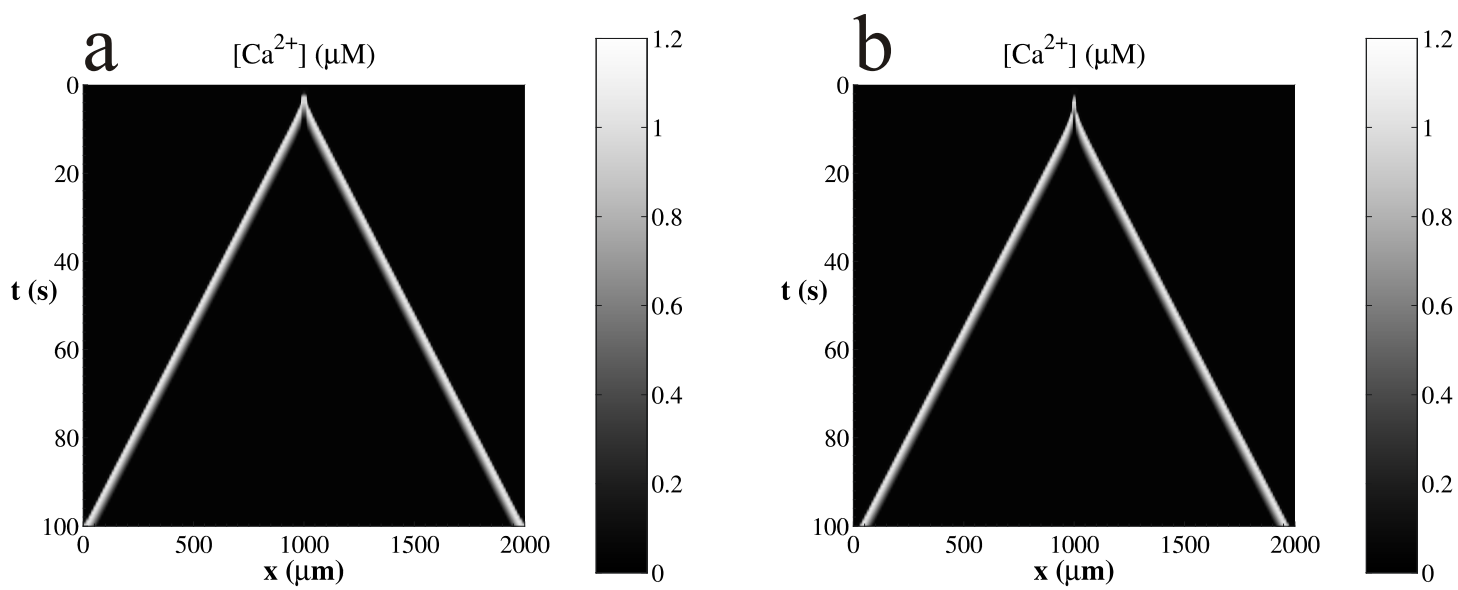

Figure S3: $\mathrm{Ca}^{2+}$ dependent ATP release: comparison of the case in which only ATP diffuses, $D_{\text {ATP }}=350 \mu \mathrm{m}^{2} / \mathrm{s}$ (panel a) with the case in which both ATP and $\mathrm{IP}_{3}$ diffuse, $\mathrm{D}_{\text {IP3 }}=280 \mu \mathrm{m}^{2} / \mathrm{s}$ (panel b). The stimulus in both cases is a square pulse in space of width $12 \mu \mathrm{m}$ and amplitude 3 $\mu \mathrm{m}$ imposed at $\mathrm{x}=1000 \mu \mathrm{m}$ at $\mathrm{t}=0 \mathrm{~s}$. The responses are qualitatively the same, quantitatively they differ only slightly. 

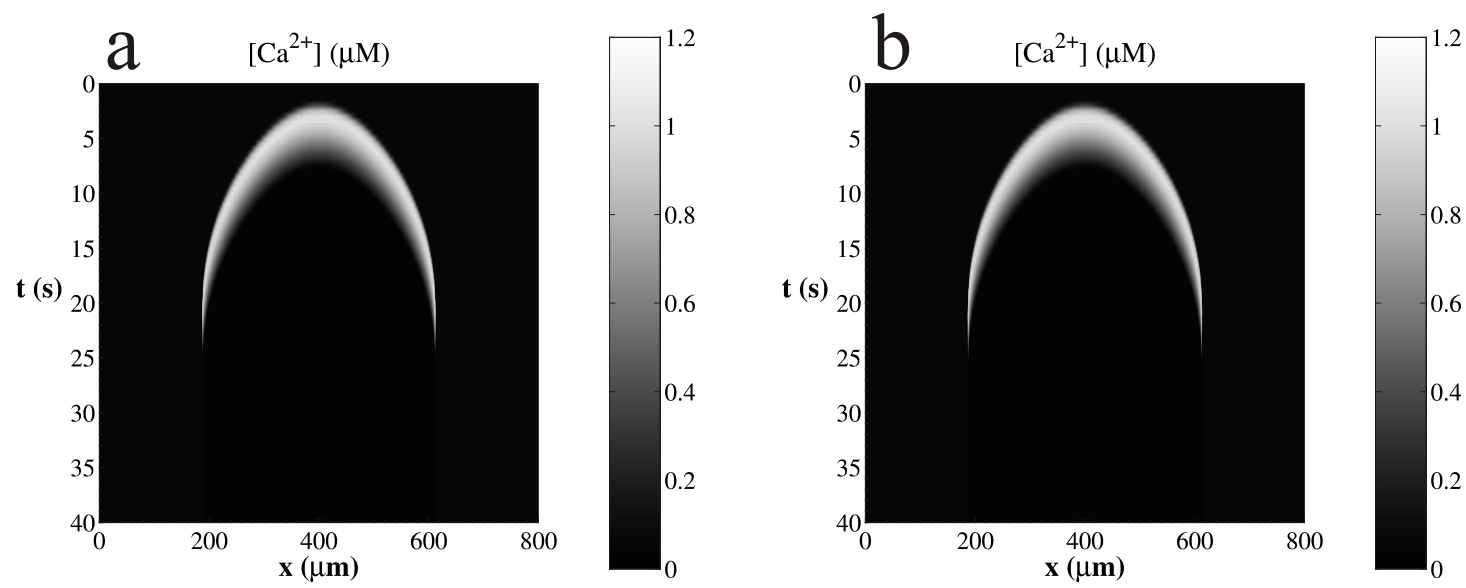

Figure S4: $\mathrm{IP}_{3}$ dependent ATP release: comparison of the case in which only ATP diffuses, $\mathrm{D}_{\mathrm{ATP}}$ $=350 \mu \mathrm{m}^{2} / \mathrm{s}$ (panel a) with the case in which both ATP and $\mathrm{IP}_{3}$ diffuse, $\mathrm{D}_{\mathrm{IP} 3}=280 \mu \mathrm{m}^{2} / \mathrm{s}$ (panel b). The stimulus in both cases is a square pulse in space of width $15 \mu \mathrm{m}$ and amplitude $500 \mu \mathrm{m}$ imposed at $\mathrm{x}=1000 \mu \mathrm{m}$ at $\mathrm{t}=0 \mathrm{~s}$. The responses are nearly indistinct. 